\title{
THE
}

1998

\section{Design of a Multiply Nested Primitive Equation Ocean Model}

Isaac Ginis

University of Rhode Island, iginis@uri.edu

R. A. Richarson

University of Rhode Island

Lewis M. Rothstein

University of Rhode Island, Irothstein@uri.edu

Follow this and additional works at: https://digitalcommons.uri.edu/gsofacpubs

\section{Citation/Publisher Attribution}

Ginis, I., Richardson, R. A., \& Rothstein, L. M. (1998). Design of a Multiply Nested Primitive Equation Ocean Model. Mon. Wea. Rev., 126, 1054-1079. doi: 10.1175/1520-0493(1998)1262.0.C0;2.

Available at: https://doi.org/10.1175/1520-0493(1998)126<1054:DOAMNP>2.0.C0;2

This Article is brought to you for free and open access by the Graduate School of Oceanography at DigitalCommons@URI. It has been accepted for inclusion in Graduate School of Oceanography Faculty Publications by an authorized administrator of DigitalCommons@URI. For more information, please contact digitalcommons-group@uri.edu. 


\title{
Design of a Multiply Nested Primitive Equation Ocean Model
}

\author{
I. Ginis, R. A. Richardson, AND L. M. RothStein \\ Graduate School of Oceanography, University of Rhode Island, Narragansett, Rhode Island
}

(Manuscript received 15 April 1997, in final form 8 September 1997)

\begin{abstract}
A new multiply nested primitive equation ocean model is presented. The model employs a two-way interactive nesting technique successfully applied for many years in the Geophysical Fluid Dynamics Laboratory-NOAA hurricane prediction model. The formulation of the mesh nesting algorithm allows flexibility in deciding the number of meshes and the ratio of grid resolutions between adjacent meshes. Other advanced features include realistic coastline geometry and spatially variable grid spacing. The results of various idealized experiments indicate good performance of the nesting technique.

The most important feature of the model is the ability to combine large-scale and regional-scale predictions. The model is tested as a general circulation model (GCM) in a 3-yr spinup experiment of the large-scale circulation in the tropical Pacific Ocean. It demonstrates skill comparable to that of other recently developed GCMs. The resulting large-scale fields are then used in the nested configuration as initial conditions for simulations of the ocean response to a westerly wind burst and a tropical cyclone. Significant improvements over a coarse, singlemesh model have been achieved in resolving finescale features of the wind-induced current and temperature fields. These results highlight the importance of model resolution for realistic simulations of mesoscale ocean variability.
\end{abstract}

\section{Introduction}

The evolution of the atmosphere and ocean is determined by many processes operating over a wide range of temporal and spatial scales. One such scale includes synoptic-mesoscale atmospheric and oceanic phenomena, and covers a broad spectrum of environmental events that have lifetimes ranging from several hours up to a month or so. Meteorological examples of such events are tropical cyclones, wind bursts, squall lines, and other "spells" of unusual weather. Oceanic synoptic-mesoscale variability is dynamically analogous to atmospheric synoptic-scale phenomena and can be thought of as the internal weather of the ocean. It encompasses processes where energetic variability occurs on spatial scales characterized by the internal Rossby radius of deformation (Robinson 1983). The first internal Rossby radius in the ocean ranges from less than $10 \mathrm{~km}$ to several tens of kilometers and is thus two orders of magnitude or more smaller than the internal radius in the atmosphere.

Improvements in the prediction of some important synoptic-mesoscale atmospheric and oceanic phenomena can be made by coupling oceanic and atmospheric models (e.g., Ginis et al. 1997). However, although rapid

Corresponding author address: Dr. Isaac Ginis, Graduate School of Oceanography, University of Rhode Island, Narragansett Bay Campus, Narragansett, RI 02882-1197.

E-mail: iginis@gso.uri.edu progress is occurring in ocean forecast research, oceanographers are still somewhat behind meteorologists in their numerical prediction capabilities. This is particularly true in regard to simulations of ocean variability on the mesoscale. The small spatial scale of this variability requires very high horizontal and vertical resolutions that are difficult to achieve in realistic ocean simulations using conventional, single-mesh ocean models. With a single-mesh model, one is typically faced with a choice of a large-scale simulation, where high resolution is computationally impractical, or a regional simulation, in which boundary conditions are suspect and large-scale flow features are difficult to represent.

One avenue for improvement of ocean mesoscale forecasting tools involves the development of models that have multiply nested grid capabilities, a numerical technique that is widely used in meteorology [e.g., Zhang et al. (1986) and references therein]. A nestedgrid configuration makes it feasible to combine realistic large-scale simulations with mesoscale forecasts for selected regions. It allows improved horizontal resolution in model forecasts of smaller-scale oceanic phenomena, and better resolution of large gradients of oceanic variables, without requiring a fine grid resolution throughout the entire model domain. By including a nested structure, the large-scale flow can be simulated with a lower resolution, while a selected region can be examined at higher resolution with a model that has essentially the same physics and numerical structure in both regimes. 
The purpose of this paper is to present a new, highresolution, multiply nested primitive equation ocean model. The model is specifically designed to simulate mesoscale ocean dynamics while simultaneously resolving the larger-scale circulation. The development of this model was motivated by the international Tropical Ocean Global Atmosphere Coupled Ocean-Atmosphere Response Experiment (TOGA COARE), conducted in the warm pool (the area where the surface temperature exceeds $28^{\circ} \mathrm{C}$ ) of the western equatorial Pacific (Webster and Lukas 1992). In that experiment, a four-month-long intensive observational period, extending from early November 1992 through the end of February 1993, was embedded in an approximately 2-yr program of enhanced monitoring of the warm pool system. Some of the TOGA COARE scientific objectives are to explore the multiple-scale interactions that occur in and over the warm pool and to understand and simulate the dynamical connections between regional air-sea coupled processes and the large-scale air-sea system of the tropical Pacific. The horizontal resolution needed to accurately represent the synoptic-scale air-sea interaction in the TOGA COARE region with a high degree of realism is on the order of $10-15 \mathrm{~km}$. It is not practical, however, to use very high resolution grids over the entire tropical Pacific Ocean. Instead, a multiply nested grid configuration is developed in this study. Although the nestedgrid model presented in this paper has been developed with the TOGA COARE experiment in mind, we envision its use in a variety of studies that focus on mesoscale ocean and coupled air-sea variability.

The primary feature that distinguishes nested-grid models from one another is the nature of the interaction between adjacent grids of differing resolution. Information can be passed between the different grid scales by either one-way or two-way interaction. With a oneway interaction, the courser grid mesh (CGM) model is integrated and the information on the boundaries of the finer grid mesh (FGM) is saved. The FGM model is then integrated using the CGM forecast for boundary conditions. The inherent assumption in one-way interaction models is that the larger-scale motion determines the small-scale motion without feedback from the processes occurring within the FGM region. This is apparently not a good assumption for TOGA COARE. The nested-mesh system considered in this study belongs to a class of two-way nesting systems in which two neighboring mesh areas interact dynamically with each other. In a two-way nesting system, dynamical interaction between two adjacent domains can be achieved in various ways. A fairly common technique is to transfer information from the fine to the coarse mesh and vice versa in a narrow zone where the two meshes overlap. This kind of grid configuration is used in all of the few ocean nested-grid models developed so far [e.g., Spall and Holland (1991); Oey and Chen (1992); and Fox and Maskell (1995)]. For the overlapping grid system, after each integration of the CGM, the boundaries of the FGM are specified by interpolation (temporal and spatial) from adjacent coarse grid solution tendencies. At the conclusion of each FGM integration, the interior coincident grid points in the CGM are revised using some averaging procedure. Thus the CGM integration provides the time-dependent boundary values for the FGM, and, conversely, the predicted fine-mesh values are used to continually update the coincident coarse-mesh values.

The main difficulty in developing a two-way nesting system is a compatibility problem that exists at the interface where the two grids meet. For instance, a disturbance propagating from an FGM to a CGM may undergo false reflection back to the FGM or aliasing as it enters the CGM. These interface-generated problems may lead to numerical instabilities that can seriously affect the results over the entire domain. The overlapping nested procedure does not necessarily conserve fluxes of mass, heat and momentum at the interfaces between coarse and fine meshes [e.g., Spall and Holland (1991), Oey and Chen (1992)]. As a result, it can be applied for only relatively short time integrations, and only for those applications when exact conservation properties are not very critical (Fox and Maskell 1995).

An optimal procedure that will reduce the numerical problems at mesh interfaces should have the following properties: all resolvable waves propagate across mesh interfaces with only minimal changes in amplitude and structure, and mass, momentum, and heat exchange between the two grid systems are conserved. Because of the numerical difficulties involved in designing a nestedgrid procedure that fully satisfies these two requirements, it has yet to be implemented in ocean models. However, such a scheme was developed in meteorology by Kurihara et al. (1979) and successfully applied for many years in the Geophysical Fluid Dynamics Laboratory-NOAA hurricane prediction model (Kurihara et al. 1998). The main numerical ideas of their technique are implemented in the ocean nested-grid model described in this paper. Although the different components of their nested algorithm have, in one form or another, been published in the meteorological literature, its implementation in an ocean model has some new features due to differences in the governing equations and physics. This paper presents details of the model formulation, boundary forcing, and nesting algorithm, as well as the results of rigorous testing of the computer code in idealized and realistic ocean settings.

The rest of the paper is organized as follows: the ocean model formulation is presented in section 2 , the nested-grid procedure is discussed in section 3 , various numerical test experiments are presented in section 4, and simulations of the large-scale tropical Pacific circulation and numerical results of the ocean response to a wind burst and a tropical cyclone in the western equatorial Pacific are discussed in section 5. The paper ends with a summary in section 6 . 


\section{Model formulation}

\section{a. Governing equations}

The model is based on a primitive equation multilayered formulation. The distinguishing feature of a layered model is the treatment of the surface mixed layer as a layer from both a dynamical and thermodynamical standpoint. The mixed layer is treated as a turbulent boundary layer that exchanges momentum and heat with the atmosphere at its surface and with the thermocline by entrainment at its base. It is well mixed due to turbulent mixing and is vertically homogeneous in density. The stratified thermocline below is divided into an arbitrary number of numerical layers according to a sigma-coordinate system described in section $2 \mathrm{~d}$. The model employs the reduced gravity assumption so that the deep ocean is at rest below the active upper ocean. A schematic of the vertical structure of the model is shown in Fig. 1. Each layer has an integer level at its center and is bounded by half-integer levels. The governing equations, written in spherical coordinates, are presented below:

1) Equations of motion

$$
\begin{aligned}
\frac{\partial}{\partial t}\left(h_{k} u_{k}\right)= & -D_{k}\left(u_{k}\right)+\left(f+\frac{u_{k} \tan \phi}{a}\right) h_{k} v_{k}-h_{k} \frac{\partial P_{k}}{c \partial \lambda} \\
& +(w u)_{k+1 / 2}-(w u)_{k-1 / 2}+{ }_{H} F_{k}^{\lambda}+{ }_{V} F_{k-1 / 2}^{\lambda} \\
& -{ }_{V} F_{k+1 / 2}^{\lambda}, \\
\frac{\partial}{\partial t}\left(h_{k} v_{k}\right)= & -D_{k}\left(v_{k}\right)-\left(f+\frac{u_{k} \tan \phi}{a}\right) h_{k} u_{k}-h_{k} \frac{\partial P_{k}}{a \partial \phi} \\
& +(w v)_{k+1 / 2}-(w v)_{k-1 / 2}+{ }_{H} F_{k}^{\phi}+{ }_{V} F_{k-1 / 2}^{\phi} \\
& -{ }_{V} F_{k+1 / 2}^{\phi},
\end{aligned}
$$

where $\lambda$ is the longitude, $\phi$ is the latitude, $a$ is the radius of the earth, $c=a \cos \phi, f$ is the Coriolis parameter, $h_{k}$ is the layer thickness, $u_{k}$ and $v_{k}$ are the eastward and northward components of the current vector $\mathbf{V}_{k}$, respectively, and $w$ is the vertical velocity. Further, $(w \mathbf{V})_{k-1 / 2}$ and $(w \mathbf{V})_{k+1 / 2}$ are the momentum fluxes at the top and bottom of the layer $k$ due to mass exchange with the adjacent layers; ${ }_{H} \mathbf{F}_{k}$ and ${ }_{V} \mathbf{F}_{k+1 / 2},{ }_{V} \mathbf{F}_{k-1 / 2}$ represent horizontal and vertical diffusion, respectively. The operator $D$ denotes the twodimensional divergence

$$
D()=\frac{\partial() h u}{c \partial \lambda}+\frac{\partial() h v \cos \phi}{c \partial \phi} .
$$

The pressure gradient force $\mathbf{P}_{k}$ in the layer $k$ is written as

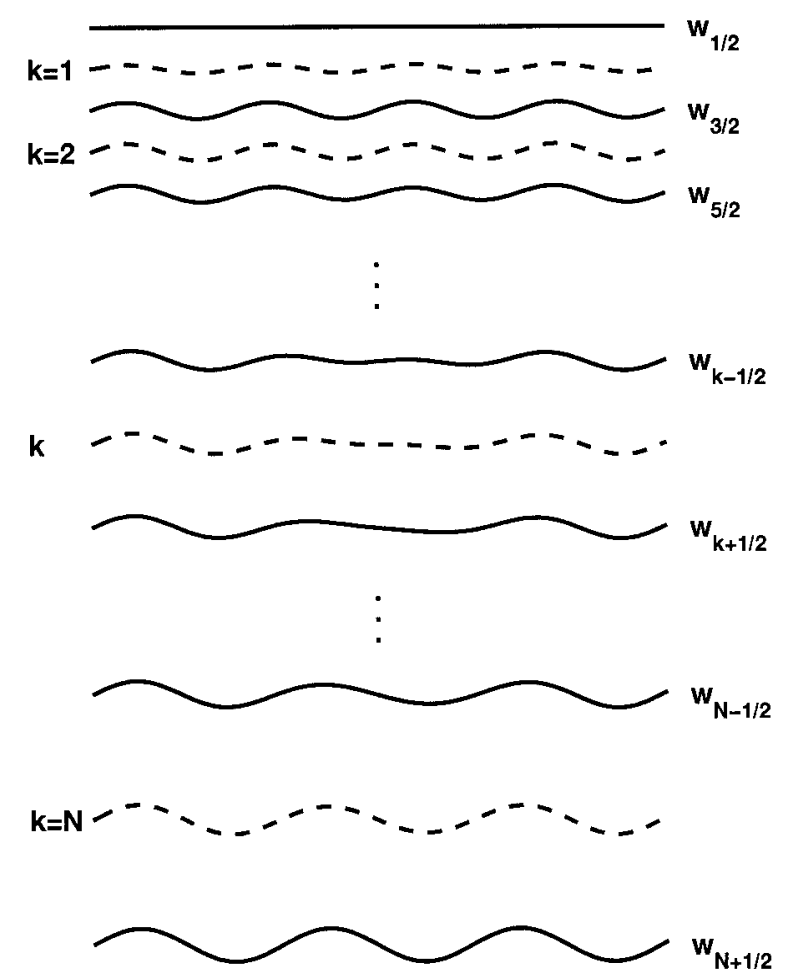

FIG. 1. Vertical model structure. Solid lines indicate layer interfaces and dotted lines indicate vertical positions of dynamical variables.

$$
\begin{aligned}
\mathbf{P}_{k}= & \left(h_{k} \frac{\partial P_{k}}{c \partial \lambda}, h_{k} \frac{\partial P_{k}}{a \partial \phi}\right) \\
= & \frac{g-b_{k}}{g} \nabla \sum_{k=1}^{N} h_{k} b_{k}+b_{k} \nabla\left(\sum_{j=1}^{k-1} h_{j}+\frac{h_{k}}{2}\right) \\
& -\nabla\left(\sum_{j=1}^{k-1} h_{j} b_{j}+\frac{h_{k} b_{k}}{2}\right),
\end{aligned}
$$

where $g$ is the acceleration due to gravity, and $b$ is the buoyancy defined as

$$
b=-g \frac{\left(\rho-\rho_{0}\right)}{\rho_{0}},
$$

where $\rho_{0}$ is a constant reference density.

2) Continuity equation

$$
\frac{\partial h_{k}}{\partial t}=-D_{k}(1)+w_{k+1 / 2}-w_{k-1 / 2},
$$

where $w_{k-1 / 2}$ and $w_{k+1 / 2}$ are the volume fluxes per unit area across the top and bottom of the layer $k$, respectively (hereafter we will refer to these fluxes as mass fluxes). 
3) Temperature and salinity equations

$$
\begin{aligned}
\frac{\partial}{\partial t}\left(h_{k} T_{k}\right)= & -D_{k}\left(T_{k}\right)+(w T)_{k+1 / 2}-(w T)_{k-1 / 2} \\
& +{ }_{H} F_{k}^{T}+{ }_{V} F_{k-1 / 2}^{T}-{ }_{V} F_{k+1 / 2}^{T} \\
\frac{\partial}{\partial t}\left(h_{k} S_{k}\right)= & -D_{k}\left(S_{k}\right)+(w S)_{k+1 / 2}-(w S)_{k-1 / 2} \\
& +{ }_{H} F_{k}^{S}+{ }_{V} F_{k-1 / 2}^{S}-{ }_{V} F_{k+1 / 2}^{S},
\end{aligned}
$$

where the second and third terms in (7) and (8) are the temperature and salinity fluxes due to mass exchange between adjacent layers. The last three terms represent the effects of horizontal and vertical diffusion of heat and salt, respectively.

The temperature and salinity obtained in (7) and (8) are used to calculate the buoyancy (5) using the UNESCO equation of state (UNESCO 1981), as adapted by Mellor (1991).

\section{b. Vertical boundary conditions}

The boundary conditions at the sea surface prescribe the fluxes that determine the air-sea exchange. Their formulation will vary for each specific simulation and may include the mass flux due to the difference between evaporation and precipitation,

$$
w_{1 / 2}=E-P
$$

momentum flux,

$$
{ }_{V} \mathbf{F}_{1 / 2}=\boldsymbol{\tau}_{0},
$$

and heat and salinity fluxes,

$$
{ }_{v} F_{1 / 2}^{T}=Q_{0}, \quad{ }_{v} F_{1 / 2}^{S}=S_{0} ;
$$

where $\tau_{0}$ is the surface wind stress vector, and $Q_{0}$ and $S_{0}$ are the surface fluxes of heat and salt in kinematic units.

At the bottom of the lowest model layer, all fluxes, $w_{N+1 / 2},{ }_{V} \mathbf{F}_{N+1 / 2},{ }_{V} F_{N+1 / 2}^{T}$, and ${ }_{V} F_{N+1 / 2}^{S}$, are set to 0 .

\section{c. Lateral boundary conditions}

The model is designed for both basin-scale and regional-scale simulations. As such it has the ability to handle "closed" land-water boundaries and "open" lateral boundaries where the computational domain is surrounded by the sea. At closed boundaries, a no-slip condition is invoked on the velocity field and there are no grid-scale or subgrid-scale normal fluxes of any quantity. For regional predictions, time-dependent boundary conditions are imposed which can accommodate either available observations or data from the integration of a larger-scale model.

When formulating open lateral boundary conditions, different types of criteria may be imposed. In this model, we implement a technique analogous to the one developed by Kurihara and Bender (1983) including im- provements made in Kurihara et al. (1989). The essence of the technique is to provide a smooth connection of the solution in the interior domain with the imposed boundary conditions. In this scheme, the open boundary condition is carried out in two steps. A prediction is first made at the boundary points using only information from the inner domain. During this step, the boundary grid values for all model variables are obtained by linear extrapolation of the data from the interior. It should be noted that, in the original Kurihara and Bender scheme, the temperatures at the boundary grid points are obtained using the thermal wind relation. However, we found that using a linear extrapolation of temperature did not create any significant imbalances of the momentum and mass fields near the boundary in our calculations. During the second step, the obtained boundary values are relaxed toward the "reference" values according to the following damping formula:

$$
\Phi_{m, b}^{n+1}=\frac{1}{1+A}\left(A \Phi_{m, b}^{n}+\Phi_{r, b}\right),
$$

where $A$ is the damping coefficient, $\Phi_{m, b}$ denotes the model boundary gridpoint values of the variable $\Phi$, and $\Phi_{r, b}$ represents the reference values.

The most important feature of the present scheme is that the reference values are not prescribed, but are obtained in the course of the model integration. The gradients, as well as the local values of the fields of the larger scale dataset, are used to define the time-dependent reference values

$$
\Phi_{r, b}=\Lambda\left(\Phi_{m, b-1}+\Phi_{0, b}-\Phi_{0, b-1}\right)+(1-\Lambda) \Phi_{0, b},
$$

where the subscript $b-1$ denotes the inner grid points next to the boundary, and $\Phi_{0}$ denotes the values from the given larger-scale data. The weight factor $\Lambda$ can take different values for different variables. Following Kurihara et al. (1989), we apply a stronger constraint on the gradient of the temperature and salinity fields $(\Lambda=$ $1)$. For momentum, however, the gradient and the velocity itself are constrained with equal weight $(\Lambda=1 / 2)$.

\section{d. Sigma-coordinate system}

The present model utilizes a sigma-coordinate system in the vertical, originally proposed by Gent and Cane (1989). The essence of the sigma coordinate is to keep the ratio of the layer depths below the mixed layer equal to a prescribed value; that is, the ratios $\sigma_{k}$ defined as

$$
\sigma_{k}=\frac{h_{k}}{\sum_{k=2}^{N} h_{k}}
$$

remain constant in the course of the model integration.

The advantage of a sigma vertical coordinate as compared to a traditional layered model is in providing high vertical resolution immediately below the mixed layer, 
where large gradients of density and velocity may occur. This interfacial (transition) layer plays a key role in the dynamics of the upper ocean because nonadvective deepening of the mixed layer occurs by entrainment of thermocline fluid across this stratified layer. Representing the character and intensity of the turbulence in the transition layer is the key to accurate modeling of the mixed layer physics. In traditional layered models, however, high resolution is difficult to achieve because of the possible vanishing of the layer below the mixed layer during entrainment.

With this vertical coordinate, the layer depths are rearranged at each model time step in the course of the model integration. As a result, mass fluxes are introduced across the layer interfaces. These mass fluxes, along with their accompanying fluxes of momentum, heat, and salt, are calculated diagnostically. At the beginning of each time step, the time tendency $(\partial / \partial t)$ of the total depth of layers 2 through $N$ is calculated first

$$
T=\frac{\partial}{\partial t} \sum_{k=2}^{N} h_{k}=-\sum_{k=2}^{N} D_{k}(1)+w_{3 / 2},
$$

where $w_{3 / 2}$ is the entrainment/detrainment velocity at the bottom of the mixed layer described in the following section. The mass flux through each layer interface is then calculated as

$$
\begin{aligned}
w_{k+1 / 2} & =w_{k-1 / 2}+D_{k}(1)+\sigma_{k} T, \\
k & =2, \quad N-1 .
\end{aligned}
$$

Associated with this mass flux, the fluxes of momentum $(w \mathbf{V})_{k+1 / 2}$, heat $(w T)_{k+1 / 2}$ and salinity $(w S)_{k+1 / 2}$ require the values of $\mathbf{V}_{k+1 / 2}, T_{k+1 / 2}$, and $S_{k+1 / 2}$. Following Gent and Cane (1989), we choose the following forms that conserve energy, heat, and salinity

$$
\begin{aligned}
\mathbf{V}_{k+1 / 2} & =\frac{1}{2}\left(\mathbf{V}_{k}+\mathbf{V}_{k+1}\right), \\
T_{k+1 / 2} & =\frac{h_{k} T_{k}+h_{k+1} T_{k+1}}{h_{k}+h_{k+1}}, \\
S_{k+1 / 2} & =\frac{h_{k} S_{k}+h_{k+1} S_{k+1}}{h_{k}+h_{k+1}} .
\end{aligned}
$$

\section{e. Parameterization of subgrid-scale processes}

\section{1) HORIZONTAL DIFFUSION}

The effects of horizontal diffusion of momentum, heat and salt are estimated by the Smagorinsky nonlinear viscosity scheme (Smagorinsky 1963). According to the scheme, the east-west and north-south components of momentum diffusion resulting from lateral stresses are computed as

$$
\begin{aligned}
& { }_{H} F_{k}^{\lambda}=\frac{\partial \tau_{k}^{\lambda \lambda}}{c \partial \lambda}+\frac{\partial\left(\tau_{k}^{\lambda \phi} \cos ^{2} \phi\right)}{a \cos ^{2} \phi \partial \phi}, \\
& { }_{H} F_{k}^{\phi}=\frac{\partial \tau_{k}^{\phi \lambda}}{c \partial \lambda}+\frac{\partial\left(\tau_{k}^{\phi \phi} \cos \phi\right)}{c \partial \phi}+\frac{\tan \phi}{a} \tau_{k}^{\lambda \lambda},
\end{aligned}
$$

where $\tau_{k}^{\lambda \lambda}, \tau_{k}^{\phi \phi}, \tau_{k}^{\lambda \phi}$, and $\tau_{k}^{\phi \lambda}$ are the stress tensors resulting from horizontal momentum mixing. These stresses are related to the tension $\left(\mu_{k}\right)$ and shearing rate of strain $\left(\nu_{k}\right)$ by

$$
\tau_{k}^{\lambda \lambda}=-\tau_{k}^{\phi \phi}=h_{k} K_{H} \mu_{k}, \quad \tau_{k}^{\lambda \phi}=\tau_{k}^{\phi \lambda}=h_{k} K_{H} \nu_{k},
$$

where $\mu_{k}$ and $\nu_{k}$ are defined as

$$
\begin{gathered}
\mu_{k}=\frac{\partial u_{k}}{c \partial \lambda}-\frac{\cos \phi}{a} \frac{\partial}{\partial \phi}\left(\frac{v_{k}}{\cos \phi}\right), \\
\nu_{k}=\frac{\partial v_{k}}{c \partial \lambda}+\frac{\cos \phi}{a} \frac{\partial}{\partial \phi}\left(\frac{u_{k}}{\cos \phi}\right) .
\end{gathered}
$$

The diffusion coefficient $K_{H}$ is determined by

$$
K_{H}=k_{0}^{2} \delta^{2}\left(\mu_{k}^{2}+\nu_{k}^{2}\right)^{1 / 2},
$$

where $\delta$ is the grid distance in the appropriate direction and $k_{0}$ is a nondimensional parameter. A value of $k_{0}^{2}$ in the range $0.1-0.2$ has been used successfully in the numerical experiments presented in this paper. Some advantages of this parameterization are that $K_{H}$ decreases as horizontal resolution improves and that $K_{\mathrm{H}}$ is small if velocity gradients are small.

Calculations of the horizontal diffusion terms in the temperature and salinity equations use the following formulas

$$
\begin{aligned}
& { }_{H} F_{k}^{T}=\frac{\partial}{c \partial \lambda}\left[h_{k} K_{H}\left(\frac{\partial T_{k}}{c \partial \lambda}\right)\right]+\frac{\partial}{c \partial \phi}\left[h_{k} K_{H} \cos \phi\left(\frac{\partial T_{k}}{c \partial \phi}\right)\right], \\
& { }_{H} F_{k}^{S}=\frac{\partial}{c \partial \lambda}\left[h_{k} K_{H}\left(\frac{\partial S_{k}}{c \partial \lambda}\right)\right]+\frac{\partial}{c \partial \phi}\left[h_{k} K_{H} \cos \phi\left(\frac{\partial S_{k}}{c \partial \phi}\right)\right] .
\end{aligned}
$$

\section{2) Vertical DifFusion}

The vertical diffusion processes in the model include three major mechanisms of vertical turbulent mixing in the upper ocean, that is, wind stirring, shear instability, and convective overturning. The parameterization of these processes is based on the hybrid mixing scheme developed by Chen et al. (1994a). It combines the advantages of a bulk mixed layer model and the Price et al. (1986) dynamical instability model. The hybrid scheme has been shown to behave more reasonably than some commonly used schemes in various applications (Chen et al. 1994a,b). We describe below the way it is implemented in the present model.

The estimate of vertical diffusion processes is first made for background diffusion by making use of the eddy diffusion coefficient technique, where the diffusivities of momentum, heat and salinity at the layer interfaces are written, respectively, as 


$$
\begin{aligned}
& { }_{V} \mathbf{F}_{k+1 / 2}=K_{M}\left(\frac{\partial \mathbf{V}}{\partial z}\right)_{k+1 / 2}, \\
& { }_{V} F_{k+1 / 2}^{T}=K_{T}\left(\frac{\partial T}{\partial z}\right)_{k+1 / 2}+I_{k+1 / 2}, \\
& { }_{V} F_{k+1 / 2}^{S}=K_{S}\left(\frac{\partial S}{\partial z}\right)_{k+1 / 2},
\end{aligned}
$$

where $K_{M}, K_{T}$, and $K_{S}$ are the vertical eddy viscosity and diffusivities of heat and salt, respectively, which are set to small constant values appropriate for background mixing. The vertical gradients of velocity, temperature, and salinity at the layer interface $k+1 / 2$ are calculated as

$$
\left(\frac{\partial \Phi}{\partial z}\right)_{k+1 / 2}=2 \frac{\Phi_{k}-\Phi_{k+1}}{h_{k}+h_{k+1}}
$$

where $\Phi$ stands for $\mathbf{V}, T$, or $S$.

The value $I_{k+1 / 2}$ in (27) is the penetrating solar radiation, written as

$$
I_{k+1 / 2}=\gamma I_{0} \exp \left(-\frac{z}{h_{\gamma}}\right),
$$

where $I_{0}$ is the solar radiation flux at the sea surface, $h_{\gamma}$ is the attenuation depth of the shortwave radiation, set to $17 \mathrm{~m}$, and $\gamma=0.33$ (Chen et al. 1994a).

Since the uppermost layer is treated as a turbulent boundary layer in the model, its deepening (shallowing) is determined by the mass exchange with the layer below, controlled by the entrainment (detrainment) rate, $w_{3 / 2}$. This rate is calculated from the following simplified bulk turbulent kinetic energy equation:

$w_{3 / 2} h_{1}\left(b_{1}-b_{2}\right)=2 m_{1} u^{* 3}-h_{1}\left(Q_{0}-m_{2} \frac{Q_{0}-\left|Q_{0}\right|}{2}\right)+Q_{1}$,

where

$$
Q_{1}=\gamma I_{0}\left[h_{1} e^{-h_{1} / h_{\gamma}}-2 h_{\gamma}\left(1-e^{-h_{1} / h_{\gamma}}\right)\right],
$$

$u_{*}=\sqrt{\tau_{0}}$ is the surface friction velocity, and $m_{1}$ and $m_{2}$ are constants set to 1.25 and 0.83 , respectively.

When the right-hand side of (30) is less than 0 , the mixed layer shallows (detrainment) and $h_{1}$ is calculated by balancing the terms on the right-hand side of (30). The detrainment velocity is then obtained as

$$
w_{3 / 2}=\frac{\Delta h}{\Delta t}
$$

where $\Delta h$ is the negative depth change, and $\Delta t$ is the time increment.

The values of $\mathbf{V}_{3 / 2}, T_{3 / 2}$, and $S_{3 / 2}$ that are necessary to estimate the fluxes of momentum, heat, and salinity associated with entrainment/detrainment are calculated using an upstream scheme:

$$
\left(\mathbf{V}_{3 / 2}, T_{3 / 2}, S_{3 / 2}\right)= \begin{cases}\left(\mathbf{V}_{2}, T_{2}, S_{2}\right), & w_{3 / 2} \geq 0 \\ \left(\mathbf{V}_{1}, T_{1}, S_{1}\right), & w_{3 / 2}<0 .\end{cases}
$$

The shear-produced mixing and convective overturning in the ocean interior are carried out diagnostically. The shear-produced mixing is parameterized using a gradient Richardson number criterion, originally introduced in Price et al. (1986). The scheme first makes estimates of the Richardson number $R_{g}$ at the layer interface between two adjacent layers $k$ and $k+1$,

$$
R_{g}=\frac{1}{2} \frac{\left(b_{k}-b_{k+1}\right)\left(h_{k}+h_{k+1}\right)}{\left|\mathbf{V}_{k}-\mathbf{V}_{k+1}\right|^{2}} .
$$

If $R_{g}$ is less than the critical value $\left(R_{c}=0.25\right)$, these two layers are partially mixed in such a way that $R_{g}$ goes back to $R_{c}$,

$$
\begin{gathered}
\Phi_{k}^{m}=\Phi_{k}-\frac{\left(1-R_{g} / R_{c}\right) h_{k+1}}{h_{k}+h_{k+1}}\left(\Phi_{k}-\Phi_{k+1}\right) \\
\Phi_{k+1}^{m}=\Phi_{k+1}+\frac{\left(1-R_{g} / R_{c}\right) h_{k}}{h_{k}+h_{k+1}}\left(\Phi_{k}-\Phi_{k+1}\right),
\end{gathered}
$$

where $\Phi$ stands for $\mathbf{V}, T$, or $S$ and superscript $m$ denotes the values after the shear-produced mixing.

The convective overturning is handled by a simple convective adjustment. When two adjacent layers $k$ and $k+1$ are statically unstable (density of the layer $k$ is greater than density of the layer $k+1$ ), the densities are mixed instantly. The new buoyancy profile is reexamined for further static instabilities and the iteration proceeds until the entire water column becomes gravitationally stable.

During the time integration of the model, the effect of vertical diffusion is taken into account in the following way. At the beginning of each time integration step, the entrainment/detrainment rate is first calculated and all turbulent fluxes are estimated at the interface between the mixed layer and thermocline $(k=3 / 2)$. That is followed by a calculation of the fluxes at the interfaces below the mixed layer resulting from the sigma coordinate layer depth rearrangement. These fluxes are used for prediction of the new values of velocity, temperature, salinity, and depth of each layer. After the completion of the time integration step, vertical mixing and convective adjustment in the ocean interior are carried out, where necessary, to determine the new vertical profiles of temperature, salinity, and velocity.

\section{Grid-nesting procedure}

\section{a. Grid configuration}

The most distinctive feature of the present model numerical design is a nested mesh system in which inner meshes of finer resolution are telescopically nested. A schematic of the nested mesh structure is shown in Fig. 2. The outermost mesh, extending to the boundary of the model domain (not shown), contains meshes of high- 


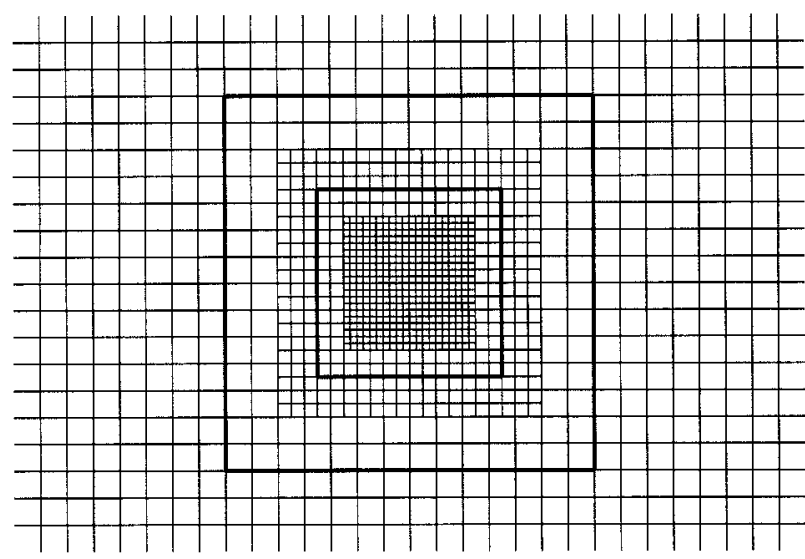

FIG. 2. A schematic of the nested mesh structure. Bold lines indicate the dynamical interfaces.

er resolution, which are telescopically nested. The diagram represents a triply nested grid configuration in which the ratio of the grid spacing between adjacent meshes is set to 2. However, the nesting strategy in our model allows flexibility in deciding the number of meshes and grid ratios for each model application. Moreover, as will be demonstrated below, the nested-grid model can be reduced to a simple single-mesh model by setting the number of grids to 1 .

The nested-grid configuration presented here belongs to the class of two-way nesting in which the time integration proceeds simultaneously for the fine and the coarse resolution domains. The most important feature of the mesh coupling procedure is that the boundary at which two neighboring domains dynamically interact (the dynamical interface, bold lines in Fig. 2) is separated from the mesh interface by a narrow zone of two coarse-grid points. This allows one to avoid simultaneously dealing with the problems resulting from grid resolution changes and dynamical coupling.

A nonstaggered grid system is used (an A grid in Arakawa's nomenclature) in which all model variables are calculated at the middle of the grid boxes. We should note here that a nonstaggered mesh is known to yield potentially large errors in the phase speeds of short gravity waves with wavelengths less than four grid intervals (Mesinger and Arakawa 1976). However, these waves are suppressed adequately in the present model through the use of a frequency selective time integration scheme and occasional spatial smoothing, methods which are described below.

\section{b. Spatial finite differencing}

Finite differencing of the governing equations is based on the box method originally developed by Bryan (1966) and Kurihara and Holloway (1967). The gradient of any quantity is computed from its value at the center of a given grid box and those on its sides. The flux divergence is estimated from the sum of fluxes across
Single stretched Grid

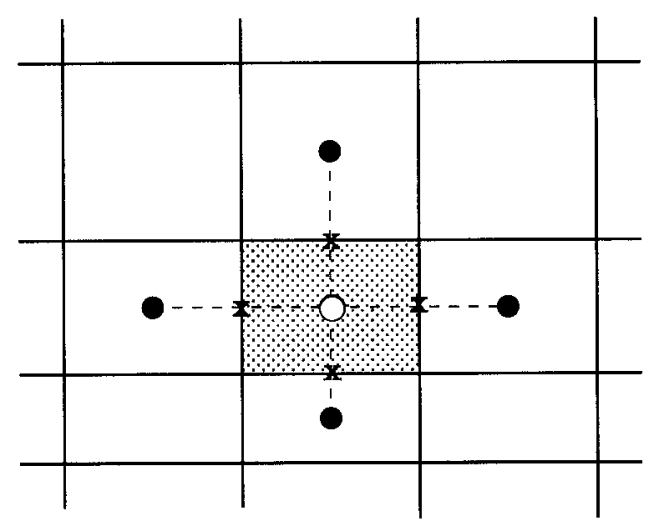

Nested Stretched Grid

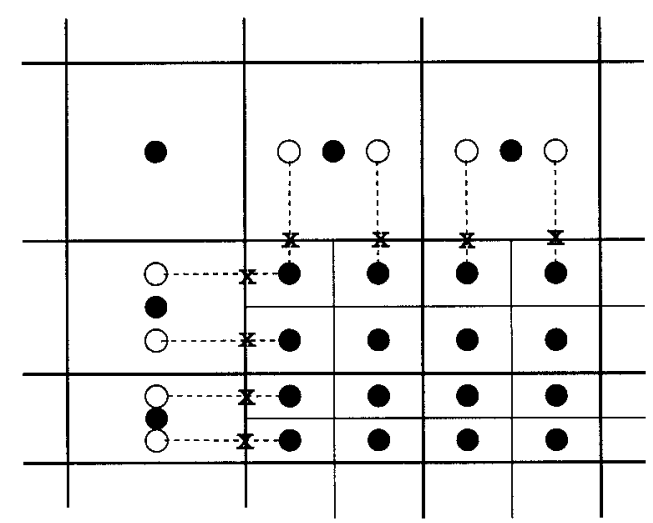

FIG. 3. Schematic illustration of the calculation of box interface values in a single but meridionally stretched mesh (upper panel) and a nested mesh system (lower panel).

the interfaces between the box and adjacent boxes. Conservation properties of mass, momentum, and energy are satisfied everywhere in this method.

In the original box method, designed for a uniformly spaced grid, the values at the box interfaces are obtained by averaging the grid values. In our model, for an irregular single-mesh or a nested configuration when a coarse box is connected to two or more finer boxes, the interface values are calculated by linear interpolation of grid values as suggested in Kurihara et al. (1979). Figure 3 illustrates how the box interface values are calculated in a single but irregular mesh and in a nested model at the interface between a CGM and FGM. In the upper part of Fig. 3, the values at the sides of the middle box (cross marks) are obtained by linear interpolation between the box grid point (open circle) and neighboring grid points (black dots). In the nested case (lower part of Fig. 3), values at the coarse grid are first interpolated 
to the auxiliary points (open circles) in the direction parallel to the interface. The values at the interface points (cross marks) are then calculated by interpolation between auxiliary points and the FGM. This method becomes identical to the original box method for a uniformly spaced single mesh.

\section{c. Time integration scheme}

The time integration is performed with a two-step iteration scheme consisting of a predictor and a corrector step (Kurihara and Tripoli 1976). The scheme is specially designed to preserve low-frequency, slow modes while suppressing high-frequency, fast oscillatory modes. The latter property is especially important for a nested-grid configuration as a large part of the noise excited at mesh interfaces usually appears as high-frequency short gravity waves. To demonstrate how the scheme is used in the present model, the equations (1), (2), (7), and (8) are expressed in the following symbolic form:

$$
\frac{\partial}{\partial t}(h \Phi)=\mathrm{LF}+\mathrm{HF}+\mathrm{DF}+\mathrm{AD}
$$

Here $\Phi$ denotes any of the variables $u, v, T$, and $S$; LF $=-D(\Phi)+\Phi D(1)$ denotes the low-frequency terms due to advection; $\mathrm{HF}=-\Phi D(1)+$ (Coriolis, metric, and pressure gradient force for $\Phi=u, v$ ) represents the terms related to high-frequency modes; and DF represents the horizontal and vertical diffusion terms and the fluxes of momentum, heat, and salt due to mass exchange between adjacent layers. The last term, AD, is included to represent the forcing effects of shear-produced vertical mixing and convective adjustment.

The time integration proceeds in two steps. During the predictor step, tentative values $\Phi_{0}^{*}$ are obtained from

$$
\frac{\Phi_{0}^{*}-\Phi^{\tau}}{\Delta t}=\mathrm{LF}^{\tau}+\mathrm{HF}^{\tau}+\mathrm{DF}^{\tau},
$$

where $\Delta t$ is the time increment and the superscript $\tau$ indicates a time level. During the corrector step, the values of $\Phi_{0}^{\tau+1}$ at the new time level $\tau+1$ are calculated first from

$$
\begin{aligned}
\frac{\Phi_{0}^{\tau+1}-\Phi^{\tau}}{\Delta t}= & {\left[(1-\alpha) \mathrm{LF}^{\tau}+\alpha \mathrm{LF}^{*}\right] } \\
& +\left[(1-\beta) \mathrm{HF}^{\tau}+\beta \mathrm{HF}^{*}\right]+\mathrm{DF}^{\tau}
\end{aligned}
$$

That is followed by the addition of the effects of shear instability and convective adjustment

$$
\Phi^{\tau+1}=\Phi_{0}^{\tau+1}+\mathrm{AD}^{\tau+1} .
$$

With the use of appropriate weights $\alpha$ and $\beta$ in (38), high-frequency modes can be suppressed while lowfrequency modes are preserved. In the numerical experiments presented in this paper, $\alpha$ and $\beta$ are set to 0.506 and 2.5 , respectively, as suggested by Kurihara et al. (1979). Using these weights, the amplitudes of high-frequency waves are strongly suppressed.

The application of the above scheme for a multiply nested computational domain is examined in detail by Kurihara et al. (1979). We only highlight here the major principles. The time integration is performed with different time increments for each mesh proceeding inward from the outermost mesh. The integration order is based on two rules: 1) integration of a given mesh proceeds only when all the inner meshes are integrated up to the time level of that mesh, and 2) when the time levels of two or more meshes are synchronized, the integration proceeds from the outermost of the synchronized meshes. For example, for a triply nested grid configuration with a ratio of $2: 1$ between mesh 1 and 2 , and a ratio of 3:1 between mesh 2 and 3, the order of integration is as follows: mesh $1,2,3,3,3,2,3,3,3$. The ratio of the time increments between neighboring meshes is the same as the ratio of the corresponding grid sizes. If the time increment for the outermost mesh is $1200 \mathrm{~s}$ in our example, those for the two inner domains will be 600 and $200 \mathrm{~s}$, respectively. With these time increments, the integration sequence brings the solutions on all grids to the same temporal location and the cycle is repeated.

\section{d. Two-way interactive procedure}

To perform the time integration of a two-way interacting, nested-grid system, special care is required at the mesh interfaces. As mentioned before, the dynamical interfaces are physically separated from the mesh interfaces in our model. The integration domains are bounded by these dynamical interfaces. This is schematically illustrated in Fig. 4. The dynamical interface shown represents the boundary between two neighboring integration domains. The shaded two-gridpoint zone, called the window frame, belongs to the integration domain of the fine mesh. Consequently, the integration of the window frame grid points is made when the fine mesh is integrated. The distinct advantage of this strategy is that the mesh interface is free from the immediate impact of the boundary conditions. Thus, noise due to dynamical coupling is kept from occurring at the mesh interface, where the change of resolution is a potential source of numerical noise.

The two-way interaction is accomplished in the following way. At a given time level, the integration begins in domain 1 (Fig. 4), taking into account the values in the window frame. During this step the fluxes and all model variables at the dynamical interface are preserved. This information is used during the integration of domain 2 to specify the boundary conditions along the dynamical interface. Since the time increment of the fine grid domain is shorter than that in the coarse-grid domain, the boundary conditions are interpolated in time, which makes the sum of fluxes during the integration of domain 2 exactly equal to the flux obtained in the integration of domain 1 [see Kurihara et al. (1979) 


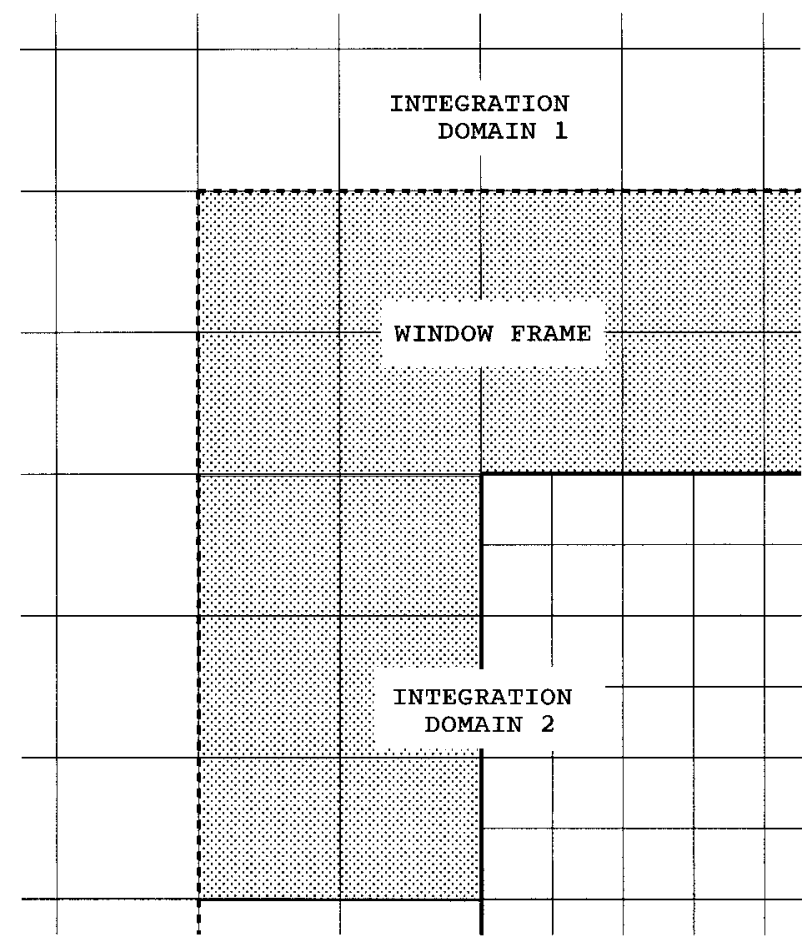

FIG. 4. Horizontal structure of two adjacent integration domains. The dynamical interface is indicated by the dashed line.

for details]. In this procedure, the transports of mass, momentum, heat, and salinity do not yield fictitious increases or decreases at the dynamical interfaces.

\section{e. Computational noise control}

High-frequency, short gravity waves are mainly suppressed by the application of a time-damping integration method. However, in a nonlinear system, stationary computational noise is inevitably generated by energy transfer along the spectra. In a real fluid, energy that is transferred to small scales is dissipated by molecular friction. A numerical solution, however, has no scales shorter than the size of a grid box and the energy therefore has a tendency to be accumulated on these scales. This may cause computational instability. Consequently, a numerical model should have a mechanism of dissipation similar to molecular friction. A common practice in numerical modeling is to apply a spatial smoothing operator, which plays the role of an artificial friction. Such an operator, however, should be used with great caution, especially in simulations concerned with gravity wave propagation. The use of too much artificial friction may lead to substantial damping of waves with longer wavelengths during removal of the short gridscale variations. For example, in a simple three-point averaging operator, sinusoidal waves with wavelengths of $4 d$ and $6 d$ (where $d$ is the grid distance) are reduced in amplitude by $50 \%$ and $25 \%$, respectively. Far more satisfactory for our present purposes is the smoothing technique introduced in Bender et al. (1993). The main idea of the method is to apply a "desmoothing" operator to a preliminary smoothed field. For illustration, a onedimensional three-point smoothing-desmoothing operator applied in the zonal direction can be written as follows:

$$
\Phi=(1-4 \kappa) \Phi_{0}+2 \kappa\left(\Phi_{W}+\Phi_{E}\right),
$$

where $\Phi_{0}$ is the center gridpoint value, and $\Phi_{W}$ and $\Phi_{E}$ are the values at the west and east interfaces between $\Phi_{0}$ and its surrounding grid points.

This operator works as a smoother when $\kappa=0.25$, but it works as a desmoother when $\kappa$ is negative and less than -0.25 . If the desmoothing operator is applied immediately following the smoothing, it will restore the amplitudes of slow-moving long waves that are reduced by the smoothing operator. When $\kappa=-0.28$, the abovementioned $4 d$ and $6 d$ sinusoidal waves have amplitudes after desmoothing only $22 \%$ and $4 \%$ smaller than the original unsmoothed amplitudes. During the model integration, we apply the smoothing-desmoothing operator sequentially in the zonal and meridional directions at appropriate time-step intervals. For the experiments discussed in this paper, this operator was applied every six time steps.

\section{f. Some programming considerations}

We have coded the model to allow for maximum flexibility in configuring different types of simulations, but, no doubt, continued improvements will be made as we apply it to new problems. The code is structured to have its basis on the coarse grid domain. Thus, given the coarse grid structure (grid distance, integration time step), a user can specify the number of inner grids, the four corners of each mesh, and the grid ratios. The program will automatically generate the grid points in every inner mesh and the time steps for inner mesh integration.

The nested system can easily be reduced to a single grid configuration by simply specifying the number of grids to be 1 . Such a feature is very desirable if one wishes to simulate both large-scale and mesoscale oceanic phenomena. One can begin the integration with a spinup of the single-mesh coarse-grid fields, interpolate the model fields onto a multinested grid configuration utilizing a routine we have developed, and then subsequently perform a mesoscale study that incorporates the large-scale features from the single grid run. This is a recipe that is followed in several of the mesoscale experiments described later. As will be demonstrated in the following section, the present model is designed to be used for simulations of the large-scale tropical Pacific Ocean circulation and for relatively short-term mesoscale simulations of ocean dynamics in specified regions. In the latter case, physical consistency between the large-scale general circulation model (GCM) and regional model is preserved. Further flexibility is provided by the option of employing variable grid reso- 
TABLE 1. Specification of the grid system in the wave propagation experiments.

\begin{tabular}{ccccc}
\hline \hline & \multicolumn{2}{c}{ Grid spacing $\left(^{\circ}\right)$} & & \\
\cline { 2 - 3 } Grid system & $\begin{array}{c}\text { East-west } \\
\text { (long) }\end{array}$ & $\begin{array}{c}\text { North-south } \\
\text { (lat) }\end{array}$ & $\begin{array}{c}\text { Domain size } \\
\text { (grid number) }\end{array}$ & $\begin{array}{c}\text { Time step } \\
(\text { s) }\end{array}$ \\
\hline Mesh 1 & $2 / 3$ & $2 / 3$ & $120 \times 60$ & 2400 \\
Mesh 2 & $1 / 3$ & $1 / 3$ & $60 \times 60$ & 1200 \\
Mesh 3 & $1 / 6$ & $1 / 6$ & $60 \times 60$ & 600 \\
\hline
\end{tabular}

lution in either the single-mesh or the multiple-mesh configurations.

\section{Numerical tests}

While the present model is designed to serve as a prediction tool for realistic ocean simulations, our immediate goal in this section is to evaluate the performance of the nesting algorithm under simplified conditions. Such tests permit comparison of the results with well-known solutions and are needed to establish the model's credibility for more complicated applications.

\section{a. Test of Kelvin and Rossby wave propagation along the equator}

We first consider Rossby and Kelvin wave propagation along the equator generated by the evolution of an initially imposed perturbation in the ocean thermocline. For these experiments, the model is simplified in two ways: the multilayer vertical structure is reduced to a single active upper layer (the second layer is at rest) and any vertical mixing or diffusion is neglected. The initial conditions assume zero velocity and a Gaussian bell-shaped perturbation in the upper-layer thickness, symmetrical about the equator

$$
h_{1}=H+\delta h \exp \left[\frac{-\left(x^{2}+y^{2}\right)}{L^{2}}\right],
$$

where $x=a\left(\lambda-\lambda_{0}\right) \cos \phi, y=a\left(\phi-\phi_{0}\right)$, and $\lambda_{0}$ and $\phi_{0}$ are the longitude and latitude of the center of the perturbation, respectively. The variables $H$ and $\delta h$ are set to 40 and $60 \mathrm{~m}$, respectively, and $L=500 \mathrm{~km}$. The values assigned for the upper- and lower-layer densities are such that the long gravity wave speed $C=$ $(g \Delta \rho H / \rho)^{1 / 2}=1.4 \mathrm{~m} \mathrm{~s}^{-1}$, so that the equatorial radius of deformation is $250 \mathrm{~km}$.

For the test experiments, we use a triply nested grid system described in Table 1. In the first run, the center of the perturbation (41) is initially placed in the middle of the finest mesh, but in the second run, it is placed outside of the two inner meshes, to the west. For the nesting algorithm to be deemed to be working well, the generated Kelvin and Rossby waves should move from the finer (coarser) meshes into the coarser (finer) meshes without any significant distortion.

Figures 5 and 6 illustrate the upper-layer depth anomalies and currents in the two runs. The initial, axisymmetric perturbation is dispersed into westwardpropagating Rossby waves and an eastward-propagating Kelvin wave. This solution is very similar to a well-known numerical solution (e.g., Philander et al. 1984). It is seen that as the waves move through the meshes of differing resolution, no numerical problems appear at the interfaces. Careful analysis of the solution indicates that the phase speed of the Kelvin wave is nearly constant as it moves from fine to coarse and coarse to fine meshes. The shape of each wave is preserved as well. Some small differences in the structure of the same waves in the coarser and finer meshes can readily be seen, however. The frontal edge of the Kelvin wave, for instance, is greatly sharpened in the finest domain. This is because the change of grid resolution from one mesh to another always results in some differences in the numerical behavior of a wave. This does not seem to cause any serious computational problem in the present tests, provided the waves are well resolved in the coarser meshes.

If the horizontal scale of the simulated waves is reduced, the solution may deteriorate as very short, highfrequency waves, unresolvable in the coarser mesh, are reflected at the mesh interface and trapped. Such difficult situations will develop inevitably in nonlinear systems and therefore must be kept under control. To demonstrate how the present model handles smaller-scale disturbances, we run two additional test experiments, which are similar to the one described above but with the horizontal size of the initial perturbation (41), $L$, reduced to 250 and $125 \mathrm{~km}$, respectively. Figure 7 shows the evolution of the depth fields in these runs. One can see that the nested-grid model works satisfactorily for smaller disturbances, as well. Because high-frequency, very short waves are effectively suppressed, no apparent wave trapping effects are observed. However, by comparing Fig. 7 and Fig. 5, one can notice that, for smaller perturbations, the wave amplitudes are substantially reduced when they move from finer to coarser meshes. This is related to an increase in numerical dispersion due to the grid-size dependency of the truncation error. If the grid resolution is not sufficiently fine relative to the scale of the disturbance, significant numerical dispersion may be generated.

This effect is further illustrated in a set of numerical experiments shown in Fig. 8. In the first run (Fig. 8a), the integration is performed using a regular, uniform 
Level Depth Anomaly
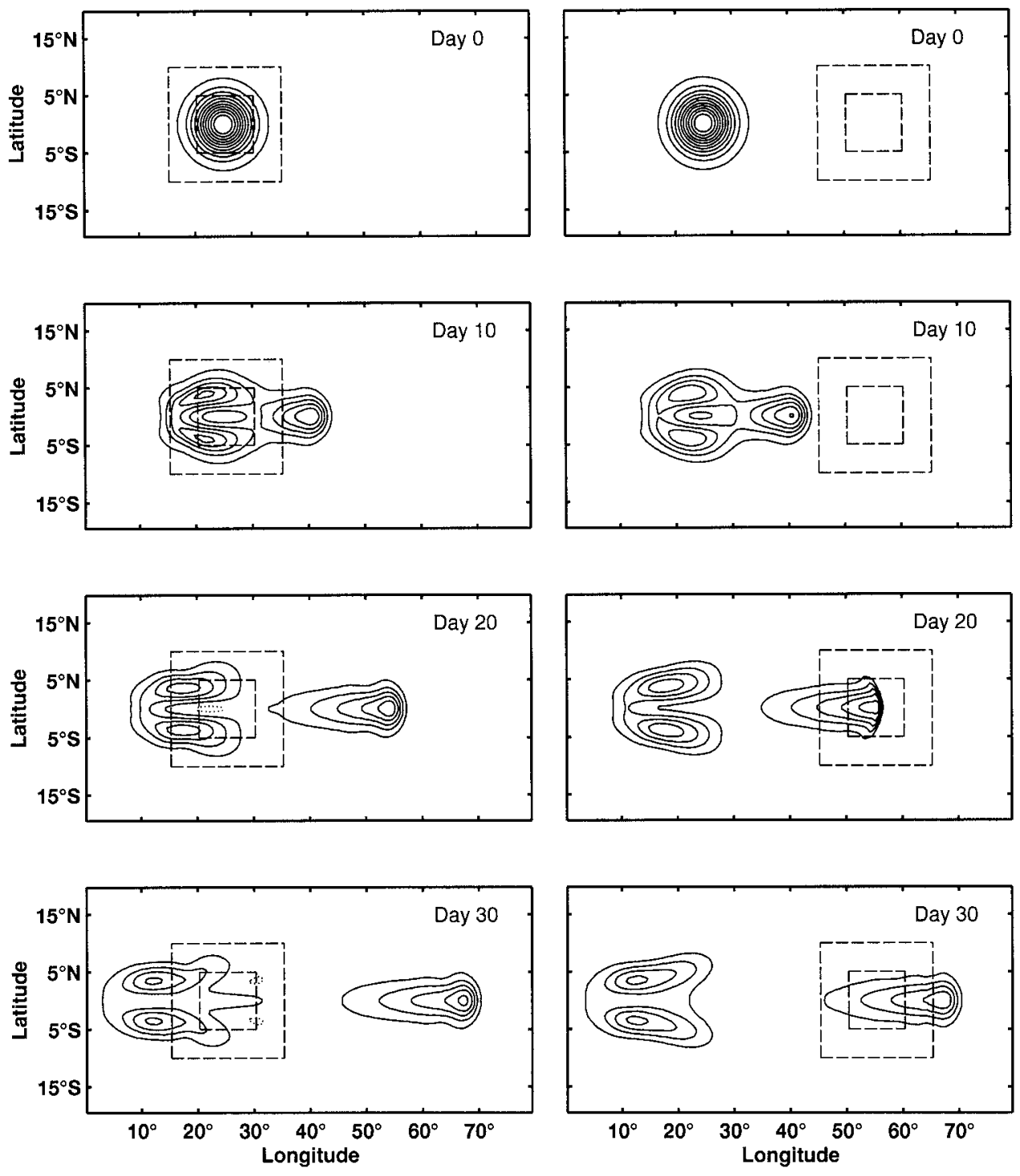

FIG. 5. Depth anomalies generated by the evolution of an initial "bell-shaped" perturbation [Eq. (41)] in two nested-grid test experiments. The grid resolutions are listed in Table 1. Contours are plotted in 2.0-m increments, with positive values shown by solid lines and negative values indicated by dotted lines. Inner grid locations are indicated by dashed lines.

grid of $1 / 6$ resolution, whereas in the second run (Fig. $8 \mathrm{~b})$, the same integration is repeated with a much coarser grid spacing of $23^{\circ}$. Note that the grid resolutions in these experiments are analogous to the resolutions of the innermost and outermost domains, respectively, in the nested experiments (see Table 1). It is evident that the depth anomalies in the latter experiment are reduced significantly. These runs can now be compared with the triply nested grid system (Fig. 8c). It is seen that the spatial structure of the depth anomalies in the innermost mesh is very similar to that in the $16^{\circ}$ resolution run, though the amplitudes are slightly smaller for the former case. This is shown more clearly in Fig. 9, where an expanded view of the innermost region of the nestedgrid experiment (Fig. 9b) is compared to the corresponding region of the $1 / 6^{\circ}$ resolution experiment (Fig. 9a). The amplitude differences may be associated with the fact that the boundary conditions for the inner domain are derived from a region of coarser resolution. Nevertheless, one sees that the finest domain captures most of the essential features of the uniformly fineresolution solution. These last comparisons illustrate the important advantage of using a multinested grid system in which high grid resolutions need be used only in the area of greatest interest without requiring high resolution over the entire computational domain. 
Surface Velocity Vectors
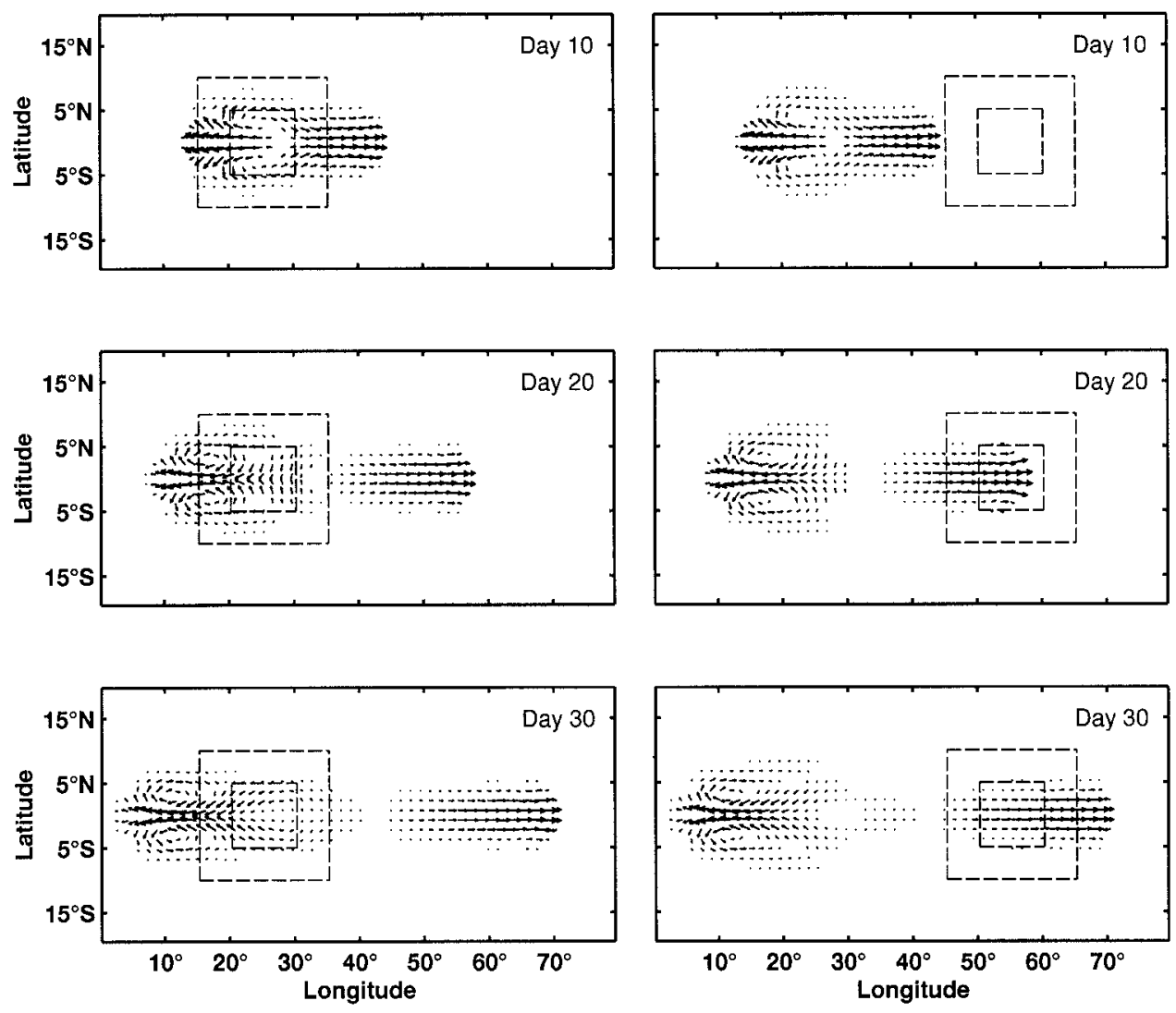

FIG. 6. Currents associated with the evolution of the initial "bell-shaped" perturbation [Eq. (41)] plotted in Fig. 5.

\section{b. Test of dipolar vortex propagation}

Another test case of the nesting procedure presented here is especially important from a computational point of view. It simulates the propagation of a dipolar vortex pair (dipole), consisting of two counterrotating eddies. These closely packed eddies are capable of trapping fluid particles and transporting them over distances much larger than their initial scale size. Thus, motion of the dipole provides a means of fluid transport, as opposed to the momentum transport that occurs during wave propagation. It yields, therefore, a rigorous test of the mass conservation conditions at the nested-mesh interfaces. The behavior of dipoles has been intensively studied analytically and numerically in geophysical fluid dynamics. Their propagation is strongly dependent upon the structure of the counter-rotating eddies (e.g., Sutyrin et al. 1994). Thus, if the simulated dipole is somewhat distorted at the mesh interfaces in the course of its propagation through the nested-grid system, the solution will quickly diverge from the reference single-mesh case.

For this test experiment, we apply the same simplified model as the one used in the previous section. The initial structure of the dipole is specified as a perturbation $\delta h$ of the upper-layer thickness $H$ as follows:

$$
\delta h=H A(r) r \sin \left(\varphi-\varphi_{0}\right),
$$

where $r$ and $\varphi$ are polar coordinates in which $r$ is the radial distance and $\varphi$ is the angle measured counterclockwise relative to eastward, $\varphi_{0}$ denotes the initial angle of dipole propagation, and $A(r)$ is a nondimensional function that determines the structure of the dipole. In the present experiment, $A(r)$ is defined using an analytical solution obtained by Larichev and Reznik (1976):

$$
A(r)= \begin{cases}1+\frac{\alpha^{2}}{k^{2}}\left[1-\frac{R J_{1}(k r)}{r J_{1}(k R)}\right], & r<R, \\ \frac{R K_{1}(\alpha r)}{r K_{1}(\alpha R)}, & r>R,\end{cases}
$$

where $J_{1}$ and $K_{1}$ denote the first-order Bessel function of the first kind and the first-order modified Bessel function of the second kind, respectively. Variable $\alpha$ is the inverse of the radius of deformation $R_{d}=(g \Delta \rho H / \rho)^{1 / 2} /$ $f$, where $H=400 \mathrm{~m}, \Delta \rho=2 \mathrm{~kg} \mathrm{~m}^{-3}$, and $f=7.27$ $\times 10^{-5} \mathrm{~s}^{-1}$. Variable $k$ is a parameter obtained from an equation ensuring continuity of vorticity at the separatrix $r=R$. Assuming that $R=3 R_{d}$, it can be shown 

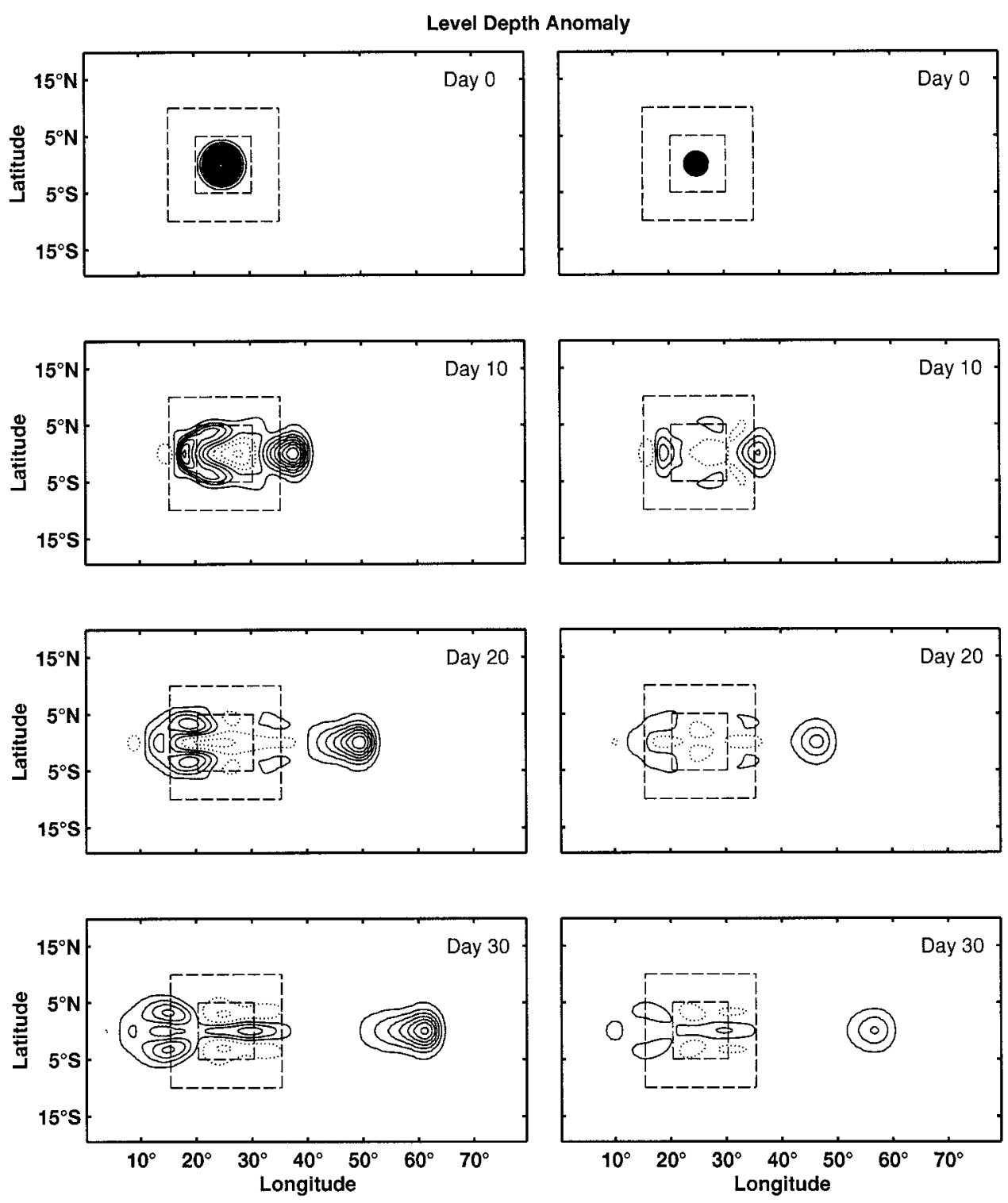

FIG. 7. The same as in Fig. 5 but with the horizontal scale of the initial perturbation reduced to $L=250$ $\mathrm{km}$ (left panels) and $L=125 \mathrm{~km}$ (right panels).

that $k=4.2 / R_{d}$. The initial velocity field is estimated from the geostrophic balance equations on an $f$ plane ( $f$ is constant).

According to the theory of dipole propagation, the symmetric dipole (42)-(43) will move along a straight line if it is on an $f$ plane (e.g., Hopfinger and van Heijst 1993). However, $f$ is variable in the present model. In this case, evolution of the dipole will be strongly dependent on its initial direction of propagation. Steady solutions exist only for dipoles initially moving in a zonal direction, either eastward $\left(\varphi_{0}=0\right)$ or westward $\left(\varphi_{0}=\pi\right)$ (e.g., Flierl 1987). For our test runs, we choose the eastward initial direction, that is, the cyclonic vortex is to the north and the anticyclonic one is to the south. According to Sutyrin et al. (1994), we should expect the dipole to move to the east with a small oscillatory excursion from the initial latitude.

Since an analytical solution is not available for the primitive equation system used here, we compare the performance of a nested grid configuration against that of a single uniform mesh. A series of preliminary test experiments with a single mesh have indicated that the dipole propagation is strongly dependent on the grid spacing. The horizontal resolution must be fine enough to resolve the structure of the eddies and internal vortex circulation that drives the self-induced dipole motion. Since our main objective here is to test the effects of mesh interfaces on the dipole propagation, we first made efforts to minimize the effects related to grid resolution changes. By reducing the grid spacing in a series of 

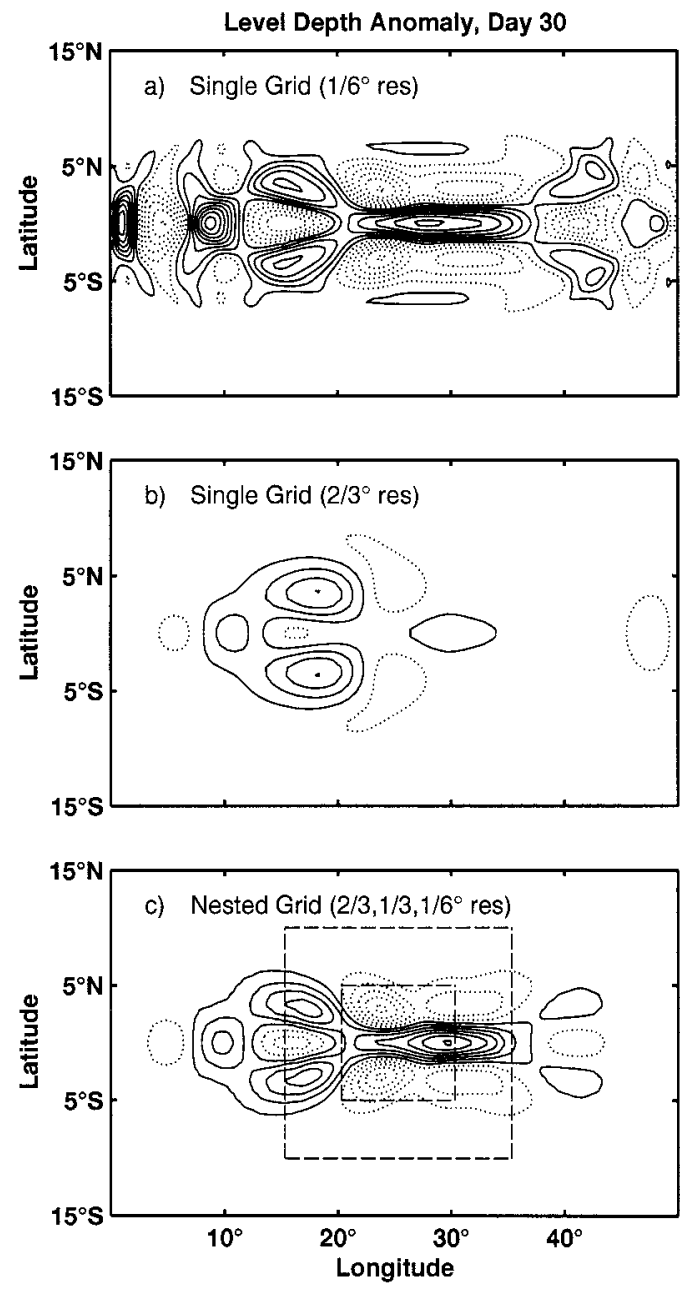

FIG. 8. Comparison of single-mesh test runs of (a) $1 / 6^{\circ}$ and (b) $2 / 3^{\circ}$ resolutions with (c) a triply nested configuration using the initial perturbation Eq. (41) with $L=250 \mathrm{~km}$. Shown here are the depth anomalies at day 30 in increments of $0.5 \mathrm{~m}$.

model runs we found that those of $0.045^{\circ}(\sim 5 \mathrm{~km})$ and $0.0225^{\circ}(\sim 2.5 \mathrm{~km})$ provided very similar solutions. We therefore applied these values to specify the corresponding grid spacing in the doubly nested grid configuration (Table 2) used for our test experiment.

Figure 10 shows the temporal development of the zonal velocity fields in the nested-grid (right panels) and uniform-grid (left panels) runs. We show here only

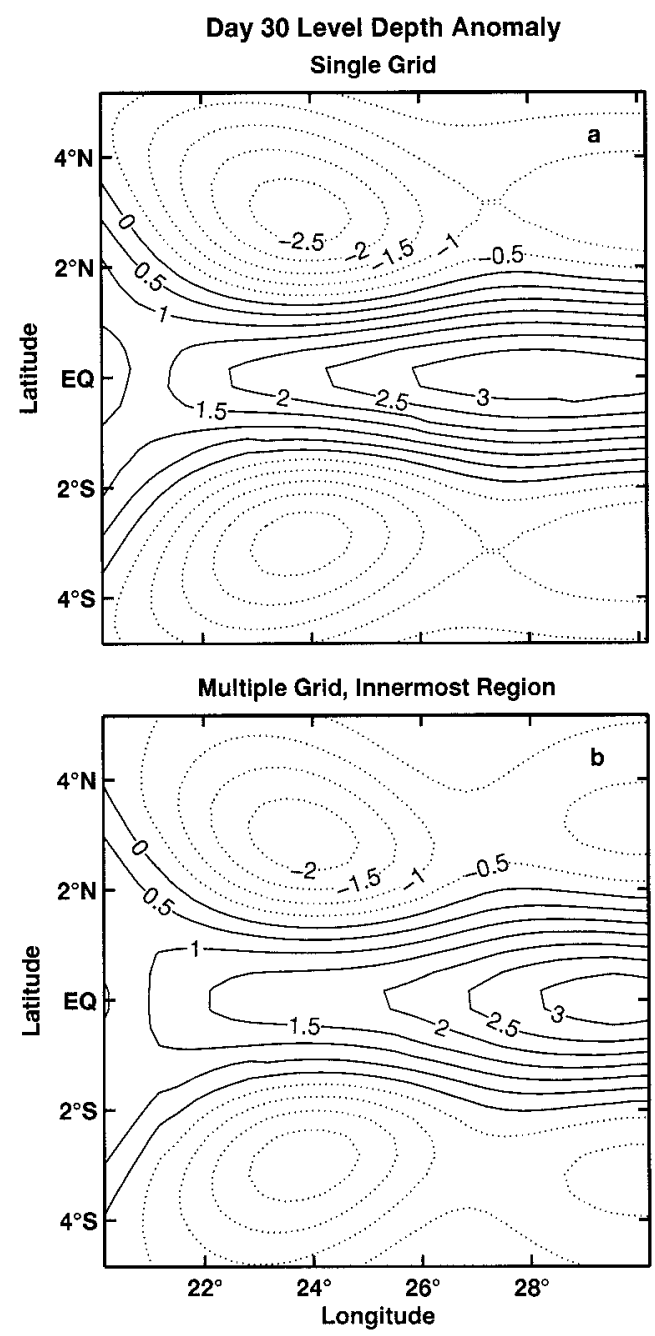

FIG. 9. Expanded view of the day 30 depth anomalies in the region from $20^{\circ}$ to $30^{\circ}$ zonally and from $5^{\circ} \mathrm{S}$ to $5^{\circ} \mathrm{N}$ meridionally. (a) The $1 / 6^{\circ}$ resolution single grid experiment, and (b) innermost mesh of the triply nested experiment.

a portion of the uniform and coarse-grid computational domains. In the beginning of the model integration, the center of the dipole was outside of the area shown in Fig. 10, located at $33^{\circ} \mathrm{N}$. During the first 30 days of dipole evolution, the anticyclonic vortex to the south became stronger than the cyclonic vortex to the north due to the differing signs of the cyclostrophic acceler-

TABLE 2. Specification of the grid system in the dipole propagation experiments.

\begin{tabular}{clcccc}
\hline \hline & & \multicolumn{2}{c}{ Grid spacing $\left(^{\circ}\right)$} & & \\
\cline { 3 - 4 } Expt. & \multicolumn{1}{c}{ Grid system } & $\begin{array}{c}\text { East-west } \\
\text { (long) }\end{array}$ & $\begin{array}{c}\text { North-south } \\
\text { (lat) }\end{array}$ & $\begin{array}{c}\text { Domain size } \\
\text { (grid number) }\end{array}$ & $\begin{array}{c}\text { Time step } \\
(\mathrm{s})\end{array}$ \\
\hline 1 & Uniform mesh & 0.045 & 0.045 & $444 \times 200$ & 400 \\
2 & Doubly nested mesh & & & & 400 \\
& Mesh 1 & 0.045 & 0.045 & $532 \times 144$ & 200 \\
\hline
\end{tabular}



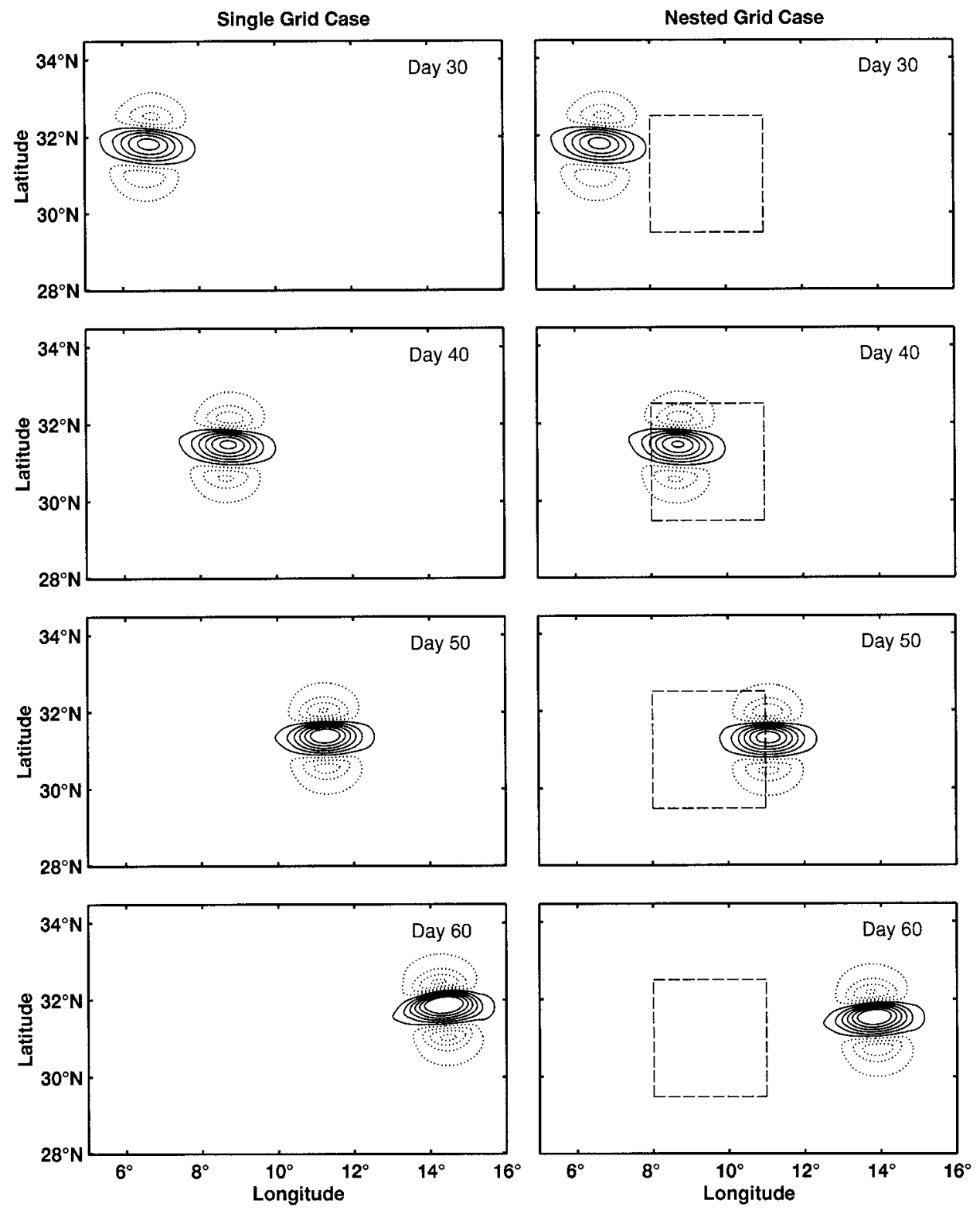

FIG. 10. Zonal velocity fields for uniform mesh (left panels) and doubly nested mesh (right panels) dipole evolution experiments. The contour interval is $30 \mathrm{~cm} \mathrm{~s}^{-1}$. Negative values are dotted.

ation terms. This caused the initial southward deflection from the eastward movement.

As the dipole continues its movement to the southeast, the cyclonic vortex strengthens while the anticyclonic vortex weakens due to the effect of conservation of absolute vorticity. As a result, the dipole reaches its minimum latitude of $31^{\circ} \mathrm{N}$ at about day 45 , and then begins moving to the northeast. The FGM in the nested system is placed in the area where the dipole reaches its most southward position but this does not cause any significant alteration in the dipole's speed of propagation as it moves through the mesh interfaces. Notice how smoothly the dipole enters the FGM and exits back to the CGM. It is important to emphasize the ability of the nested procedure to preserve the proper dipole structure, which clearly indicates very good performance of the flux conditions imposed at both the dynamical and mesh interfaces. More careful analysis of Fig. 10 shows that after the dipole has passed the FGM it begins to slightly decelerate compared to the uniform mesh experiment (the propagation speed decreases by 12\%). These changes in the dipole's motion are probably due to the higher grid resolution that the dipole experienced when it passed through the FGM. Thus, even though the dipole structure is well resolved in both meshes, small differences in the truncation error may still exist. 
TABLE 3. The $\sigma$ levels and layer depths* in the spinup experiment.

\begin{tabular}{lcccccccccc}
\hline \hline \multicolumn{1}{c}{ Level } & 1 & 2 & 3 & 4 & 5 & 6 & 7 & 8 & 9 \\
\hline$\sigma$ & - & 0.029 & 0.044 & 0.074 & 0.147 & 0.147 & 0.147 & 0.147 & 0.294 \\
Layer depth (m) & 60 & 10 & 15 & 25 & 50 & 50 & 50 & 50 & 100 \\
\hline
\end{tabular}

* The layer depth distribution is an example for the case when the average depth of the simulated upper ocean is $400 \mathrm{~m}$ and mixed layer depth is $60 \mathrm{~m}$.

In summary, the results presented in this and the preceding section suggest that the impact of mesh nesting on Rossby and Kelvin wave propagation and dipole evolution was minimal. However, these test experiments were performed with a simplified model without the effects of horizontal and vertical eddy diffusions. For the full model, the noise level can be expected to be higher than it was in these simple cases. The performance of the nested grid scheme has to be examined by applying it to the full model. Such experiments are presented in the following sections.

\section{Examples of realistic ocean simulations}

While detailed real-case applications of this nestedgrid model will appear in subsequent publications, in this section we demonstrate its general skill in a realistic ocean environment. Our main objective here is to evaluate the robustness and usefulness of the nested-grid technique and to illustrate what one might learn of the detailed mesoscale processes that are resolved in the finer meshes.

\section{a. Simulation of the tropical Pacific circulation}

In this section we will demonstrate the model performance as a GCM, presenting the results of a large-scale simulation of the tropical Pacific ocean. For this simulation, a single but variable-resolution mesh configuration will be used. Although the major components of the model physics, vertical sigma coordinate, and hybrid mixing scheme have already been successfully tested for largescale tropical Pacific simulations by Gent and Cane (1989), Chen et al. (1994a), and Chen et al. (1994b), the present model is fundamentally different in its numerical realization. Additionally, our model differs by the implementation of the Smagorinsky nonlinear viscosity scheme for horizontal diffusion. The purpose of the present simulation is twofold: to establish the model's credibility in realistically reproducing the main features of the large-scale tropical Pacific circulation and to provide initial conditions and reference runs for comparisons with the nested model.

In the experiment discussed here, the computational domain covers the whole tropical Pacific region and has 166 longitude grid points spanning from $124^{\circ} \mathrm{E}$ to $70^{\circ} \mathrm{W}$ and 92 latitude grid points from $30^{\circ} \mathrm{S}$ to $30^{\circ} \mathrm{N}$. The grid spacing is a constant $1^{\circ}$ in longitude but is variable in latitude, with a resolution of $\frac{1}{3}$ near the equator, increas- ing poleward in such a way that it is about $1^{\circ}$ at $20^{\circ} \mathrm{N}$ (S). Global topography data at $16^{\circ}$ resolution, prepared by the U.S. Navy's Fleet Numerical Oceanography Center, are used to determine the presence of land. The resulting coastline is then smoothed somewhat to avoid numerical instabilities and islands are eliminated. The upper ocean is divided into nine levels. Positions of these levels in the sigma coordinate are listed in Table 3. Note that the highest resolution is right below the mixed layer, that is, an important region for vertical turbulent mixing is well resolved.

The model is initialized with annual mean Levitus (1982) temperature and salinity. The thickness of the upper mixed layer is calculated based on a vertical gradient of density criterion. The $1027 \mathrm{~kg} \mathrm{~m}^{-3}$ density level is chosen as the model base and, with the total thermocline depth thus determined, the thickness of each layer is calculated according to the sigma coordinate.

The horizontal boundary conditions in this run are nonslip and nonflux at all boundaries. However, near the northern (southern) boundary of the domain, poleward of $25^{\circ} \mathrm{N}(\mathrm{S})$, temperature and salinity are gradually relaxed toward climatology. The model starts from rest and is forced by annual mean climatological surface wind stress and heat fluxes. The wind stress is derived from the Florida State University (FSU) pseudostress climatology. The net heat flux is parameterized according to Gordon and Corry (1991)

$$
Q_{0}=Q\left(\mathrm{SST}_{c}\right)+\lambda\left(\mathrm{SST}_{c}-T_{1}\right),
$$

where $Q\left(\mathrm{SST}_{c}\right)$ is the climatological heat flux at the Levitus annual mean sea surface temperature $\left(\mathrm{SST}_{c}\right), T_{1}$ is the upper mixed layer temperature predicted by the mod$\mathrm{el}$, and $\lambda=34.2 \mathrm{~W} \mathrm{~m}^{-2}{ }^{\circ} \mathrm{C}^{-1}$ is the relaxation parameter.

The heat flux $Q\left(\mathrm{SST}_{c}\right)$ is calculated using the simplified bulk formula of Seager et al. (1988), with annual mean cloud cover from the International Satellite Cloud Climatology Project (Rossow and Schiffer 1991). We use the total cloud fraction to determine the heat flux, making no distinction between high, middle, and low clouds. This introduces some inaccuracies to the heat flux calculation and a small correction is applied to avoid excessive surface heating in some locations, most notably near the northern Central American coast. The Seager et al. (1988) algorithm also requires wind speed for the heat flux calculation. This speed was derived from the FSU wind stress assuming a surface drag coefficient of $1.5 \times 10^{-3}$. The second term on the right-hand side of (44) indicates a relaxation back to climatology on a timescale of 20 

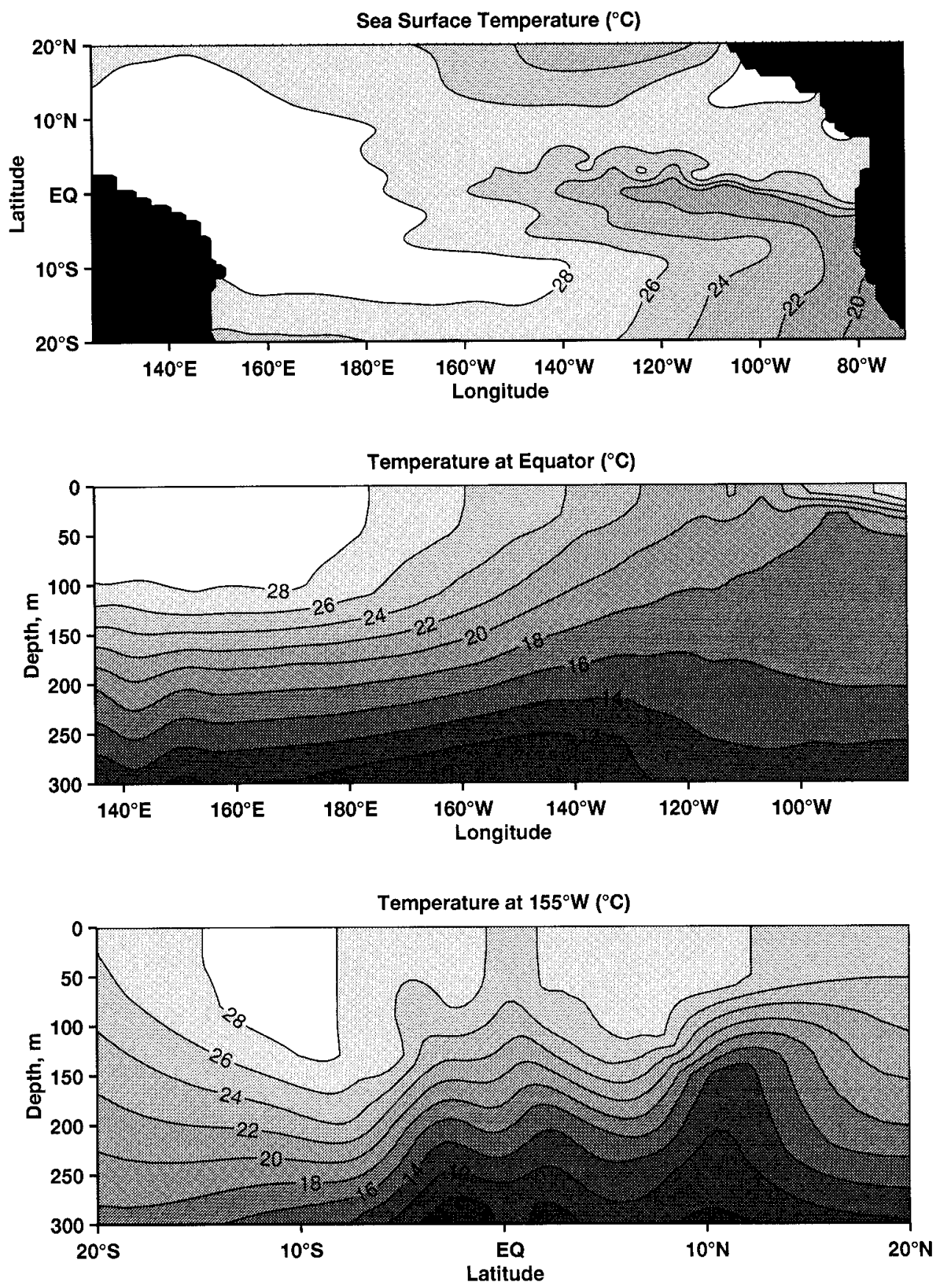

FIG. 11. Temperature fields after 1080 days for the large-scale equatorial circulation in the Pacific Ocean using a single-mesh variable grid configuration.

days for a mixed layer of $30 \mathrm{~m}$ deep. This is necessary to avoid excessive model drift from climatology when forced with constant annual mean fluxes. The applied forcing is certainly simplified and not very realistic. However, our purpose here is not to simulate real data, but rather to reproduce the major components of the tropical Pacific circulation and compare the results with other GCM runs utilizing similar forcing, in particular, with the Chen et al. (1994a) study.
The model is integrated for three years. This time integration is sufficient to establish a quasi-equilibrium state in the present experiment. Figure 11 shows snapshots of horizontal and vertical temperature distributions at day 1080 . The overall temperature field shows many characteristics that are well known from observations and other model simulations such as the warm pool in the west and the cold tongue in the east. The zonal cross section displays an increase in the thermocline depth from 

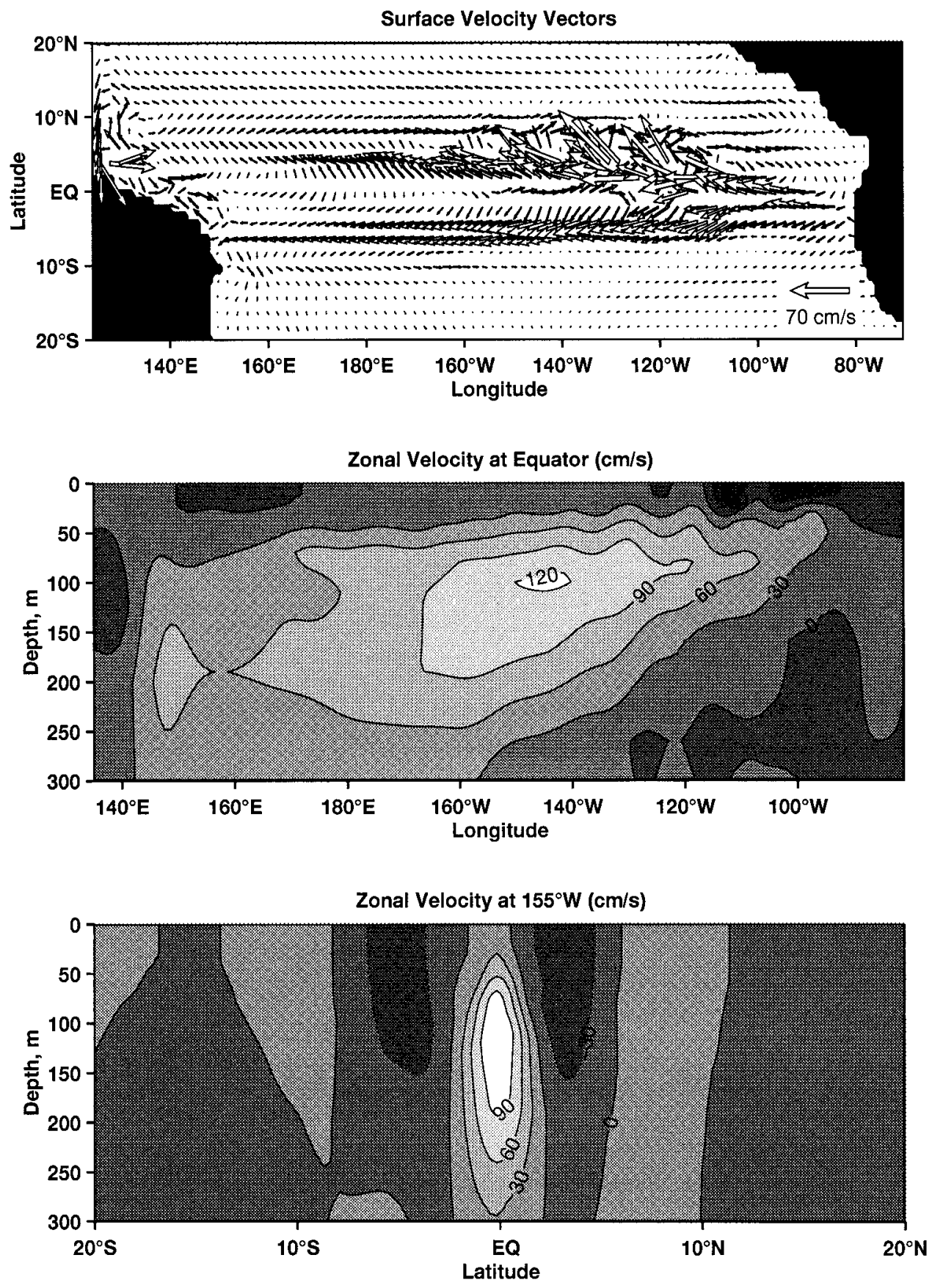

FIG. 12. The same as in Fig. 11 but for velocity fields.

east to west along the equator, which is indicative of the general east-west pressure gradient that balances the mean wind stress and, at the same time, produces and maintains the eastward-flowing Equatorial Undercurrent (Fig. 12). The meridional cross section along $155^{\circ} \mathrm{W}$ displays, for instance, the well-known ridge-trough structure in the tropical and subtropical region observed in the Hawaii-Tahiti Shuttle experiment (Wyrtki and Kilonsky 1984). The equatorial trough is located on or slight- ly south of the equator, the equatorial ridge near $3^{\circ} \mathrm{N}$, the countercurrent trough at $6^{\circ}-7^{\circ} \mathrm{N}$ and the North Pacific ridge along $12^{\circ}-13^{\circ} \mathrm{N}$. These structures are well correlated with the simulated tropical surface currents (Fig. 12), that is, the North Equatorial Current runs between the North Pacific ridge and the countercurrent trough, and the North Equatorial Countercurrent between the equatorial ridge and the countercurrent trough. More detailed analysis of the results is beyond the scope of the 


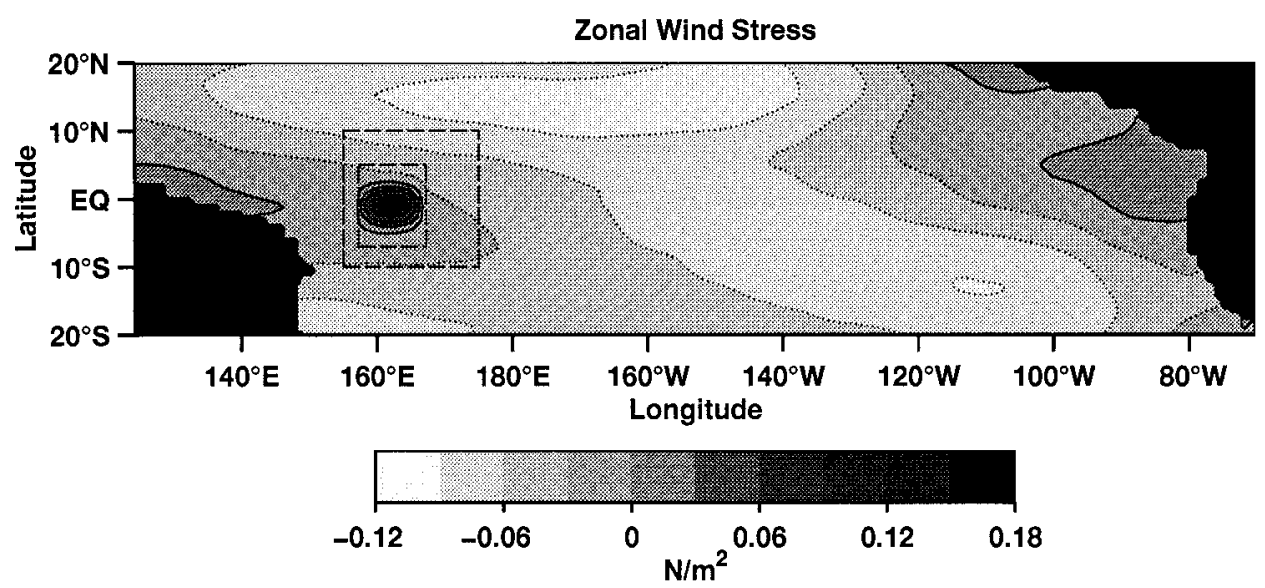

FIG. 13. Zonal wind stress and the triply nested grid structure for the WWB experiment.

present work. However, it is important to emphasize that this simulation is in very good agreement with the Chen et al. (1994a) study, indicating that their hybrid vertical mixing scheme is successfully implemented into the present model.

One of the most notable features of the simulated temperature and velocity fields is the presence of equatorial instability waves in the eastern Pacific, which are detectable all the way to the date line. These cause SST fluctuations as large as $2^{\circ} \mathrm{C}$ at $2^{\circ}-3^{\circ} \mathrm{N}$. It appears, however, that appearance of the instability waves in the model solution is very sensitive to the choice of the Smagorinsky constant, $k_{0}^{2}$ in (23). A value of $k_{0}^{2}=0.1$ is used for the present simulation. In a series of numerical experiments, we found that larger values lead to substantial weakening of these waves and their eventual disappearance for $k_{0}^{2}=1$.

The successful model performance in reproducing the major features of the large-scale equatorial Pacific circulation is quite comparable with other advanced GCMs recently developed for the tropical Pacific Ocean [e.g., Chen et al. (1994a), Zhang and Endoh (1992), Philander et al. (1987)]. However, the present model has a unique characteristic that other models do not typically possess: the capability to implant nested meshes of higher resolution in any specified area of potential interest. The model skill in simulating regional air-sea interactions in the presence of realistic large-scale circulation is evaluated in the next two sections.

\section{b. Wind burst forcing in the western equatorial Pacific}

The nested-grid model presented here is specifically tailored to perform realistic simulations of the oceanic response to synoptic-scale wind forcing and its impact on the low-frequency evolution of the coupled oceanatmosphere system. A prominent example of such forcing is the phenomenon of surface westerly wind bursts (WWB) in the western equatorial Pacific warm pool. It has been speculated that WWB's may be an important component of the El Niño-Southern Oscillation (ENSO) cycle (Harrison and Giese 1991). These short-duration episodes of westerly winds, often lasting for a few days, with typical horizontal scales from a few hundred to one thousand kilometers, have a significant effect on the upper-ocean equatorial current, salinity, and temperature structure. Understanding and predicting the oceanic response to WWB forcing have been major objectives of TOGA COARE.

For a test experiment, we use an idealized representation of a WWB, described here as a stationary narrow wind anomaly imposed just south of the equator,

$$
\tau_{0}^{\lambda}=\tau_{\max }^{\lambda} e^{-\left[\left(\lambda-\lambda_{0}\right)^{2} / L^{2}\right]} \sin \left(\pi \frac{\phi-\phi_{1}}{\phi_{2}-\phi_{1}}\right),
$$

where $\tau_{\max }=0.2 \mathrm{~N} \mathrm{~m}^{-2}$, and $L=2.5^{\circ}$. This anomaly is added to the annual mean climatological wind in the region of the TOGA COARE field experiment: $\phi_{1}=$ $157^{\circ} \mathrm{E}, \phi_{2}=167^{\circ} \mathrm{E}$, and $\lambda_{0}=1^{\circ} \mathrm{S}$.

In order to better resolve the spatial structure of the WWB and the resulting oceanic response, we introduce a triply nested configuration in which the meshes of higher resolution are placed in the area of wind burst forcing, as shown in Fig. 13. The outermost mesh covers the entire tropical Pacific region and has the same geometry as the computational domain in the previously described spinup run. The locations of the two inner meshes are chosen to approximately match the outer sounding array (OSA) and large-scale domain (LSD) domains of TOGA COARE (see Webster and Lukas 1992). The parameters of the nested-grid system are listed in Table 4. Note that the grid spacing is set to be uniform within each mesh in this test; however, this model also allows the flexibility of embedding finer meshes into a coarse domain with variable grid spacing. The latter option has been successfully tested but the results are not presented in this paper.

In these runs, we keep the structure of vertical layers the same as listed in Table 3. The nested model is ini- 
TABLE 4. Specification of the grid system in the WWB experiment.

\begin{tabular}{|c|c|c|c|c|}
\hline \multirow[b]{2}{*}{ Grid system } & \multicolumn{2}{|c|}{ Grid spacing $\left({ }^{\circ}\right)$} & \multirow[b]{2}{*}{$\begin{array}{l}\text { Domain size } \\
\text { (grid number) }\end{array}$} & \multirow[b]{2}{*}{$\begin{array}{c}\text { Time step } \\
\text { (s) }\end{array}$} \\
\hline & $\begin{array}{l}\text { East-west } \\
\text { (long) }\end{array}$ & $\begin{array}{l}\text { North-south } \\
\text { (lat) }\end{array}$ & & \\
\hline Mesh 1 & $1 / 2$ & $1 / 2$ & $320 \times 120$ & 2400 \\
\hline Mesh 2 & $1 / 4$ & $1 / 4$ & $76 \times 76$ & 1200 \\
\hline Mesh 3 & $1 / 8$ & $1 / 8$ & $76 \times 92$ & 600 \\
\hline
\end{tabular}

tialized using the fields resulting from the 3-yr singlemesh model spinup run discussed above. The gridpoint values of each variable in the nested domain are obtained from the neighboring coarse gridpoint values using twodimensional bilinear interpolation. The climatological fluxes at the sea surface are interpolated in the same manner. Note that, in future model applications, for cases in which additional finer-resolution data are available, these data can be used to enhance the description of the initial fields in the inner meshes.

In a preliminary test experiment, the nested model was first run with no WWB forcing. This run represents a mere continuation of the spinup run but with the nested meshes introduced. Figure 14 shows temperature and velocity vector fields after 30 days of integration that are compared to a reference single-mesh run. The horizontal resolution in the latter run is uniform $12^{\circ}$, and equal to the resolution of the outermost mesh in the nested model. The area shown in Fig. 14 is for the region occupied by the two inner meshes. Examination of the model fields reveals no distortions in the vicinity of the interfaces, which are frequently the site of spurious wave disturbances in nested-grid models with ill-posed interface conditions. The gross characteristics of the fields are quite similar in the nested and reference runs. However, use of the nested model has allowed the development of steeper horizontal temperature gradients, particularly immediately north of the equator. Stronger westward flow associated with the North Equatorial Current has developed in the nested run. Smaller-scale variability in the velocity field is also evident in the finest mesh, which is not resolved in the reference model. Outside of the second mesh region, the results for the nested and single-mesh runs are quite similar (not shown), although some differences exist near the mesh interface. These differences are due to finescale information that is fed back from the finer meshes and that then propagates outward to the coarse domain.

In the WWB experiment, the wind anomaly (45) was impulsively applied at day 1090 and remained in effect for 10 days. The integration was then continued for another 20 days to simulate the relaxation of the burstinduced circulation. Figure 15 shows zonal velocity fields in the region of the two inner meshes of the nested-grid model. These are compared with those for the singlemesh model of $1 / 2^{\circ}$ resolution, shown for the same region. The dynamical response to WWB forcing initially involves a meridionally convergent, eastward-accelerating
Yoshida jet (Yoshida 1959) in the surface layer. In the nested model, this jet reaches a speed of about $110 \mathrm{~cm}$ $\mathrm{s}^{-1}$ at day 1100 because the Coriolis force is ineffective at retarding and deflecting currents near the equator. The wind burst also generates inertia-gravity waves and mixed Rossby-gravity waves that cause the surface jet to deviate toward the equator. In the single-mesh run, the jet is weaker and appears to be wider in the meridional direction, indicating that the grid resolution is not sufficient to resolve its structure well. At later times in the evolution of the current field, equatorial Kelvin and long Rossby waves excited at the zonal extremes of the wind burst propagate into the WWB forced region and lead to deceleration of the eastward jet. By day 1110 the jet is clearly split into two packets. One moves to the west and is associated with the propagation of a Rossby wave and the other, moving to the east, is associated with a Kelvin wave. It is evident that the wave structure in the nested run is significantly more detailed than in the single-mesh run. Smaller-scale variability is seen throughout the region occupied by the fine mesh. Encouragingly, there is still no noticeable noise or distortion of the fields at the mesh interface. At day 1120, another important difference in the wave evolution can be observed: in the singlemesh run, the Rossby wave is leaving the area with significantly faster speed. This results from the fact that the wave phase and group velocities have been affected by the grid resolution. Thus, an apparent improvement in the representation of the ocean response to the wind burst is achieved in the nested run.

Figure 16 shows zonal and meridional cross sections of the zonal current at day 1100. Prior to the onset of the WWB, the ocean circulation is characterized by a weak westward current of about $5 \mathrm{~cm} \mathrm{~s}^{-1}$ in the surface layer and the eastward equatorial undercurrent, with a maximum of $40 \mathrm{~cm} \mathrm{~s}^{-1}$, in the thermocline at depths of 150-175 m (Fig. 11). The WWB-driven eastward jet is characterized by upwelling at the western edge of the wind burst and downwelling at the eastern edge and strong vertical shear across the mixed layer base due to vertical turbulent mixing and downward advection of surface momentum. Strong acceleration of the surface flow is accompanied by rapid deceleration of the Equatorial Undercurrent in the upper thermocline. This kind of behavior is in qualitative agreement with some observations of the ocean response to a wind burst (e.g., McPhaden et al. 1992) and more detailed numerical solutions (Zhang and Rothstein 1998). The nested- and 

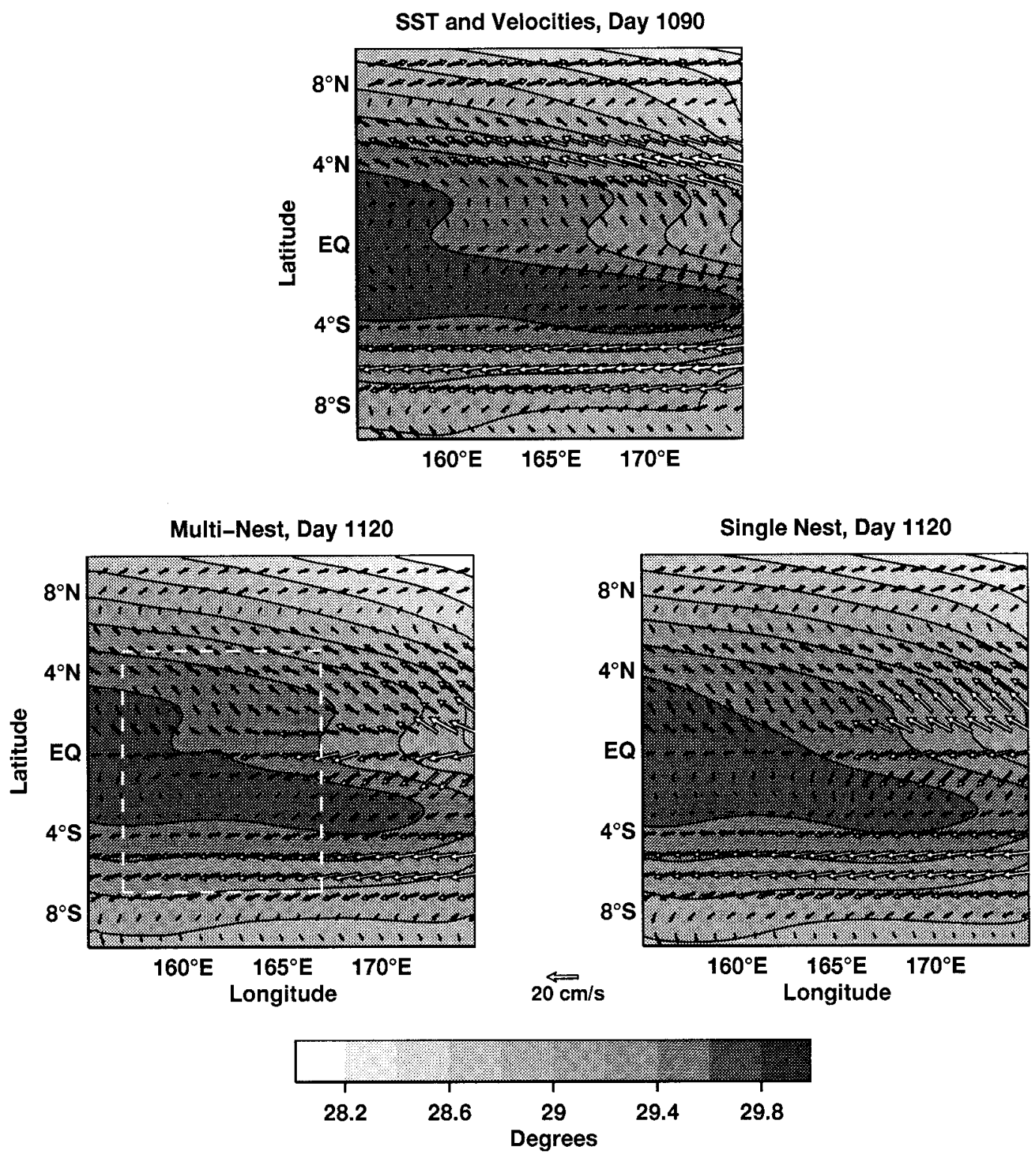

FIG. 14. Test experiment with no WWB forcing applied. Surface temperature and velocity fields are plotted in the region corresponding to the two inner meshes in the nested model. Initial fields at day 1090 (top), fields at the end of the nested run (left), and at the end of the single-mesh run (right) are shown.

single-mesh runs are qualitatively quite similar, but some differences in the velocity structure are readily observed, especially in the finest-mesh region. For example, in the nested run, the velocity shear and mixed layer deepening near the eastern edge of the WWB reach a depth of $100 \mathrm{~m}$, compared to about $75 \mathrm{~m}$ in the single mesh. The structure of the subsurface currents is noticeably different as well. Thus, although the two runs have the same high vertical resolution in the upper ocean, use of the nested model has substantially improved the simulation of the vertical structure of the wind-generated velocity fields. The implication of this result is quite important. It indicates that in order to achieve better simulations of the upper-ocean structure during synoptic-scale wind forcing, it is not sufficient to have high vertical resolution, but high horizontal resolution as well.

\section{c. Ocean response to real-case tropical cyclone forcing}

In the last test experiment presented in this paper we illustrate the ability of the nested model to simulate the ocean response to a tropical cyclone in the western $\mathrm{Pa}$ - 

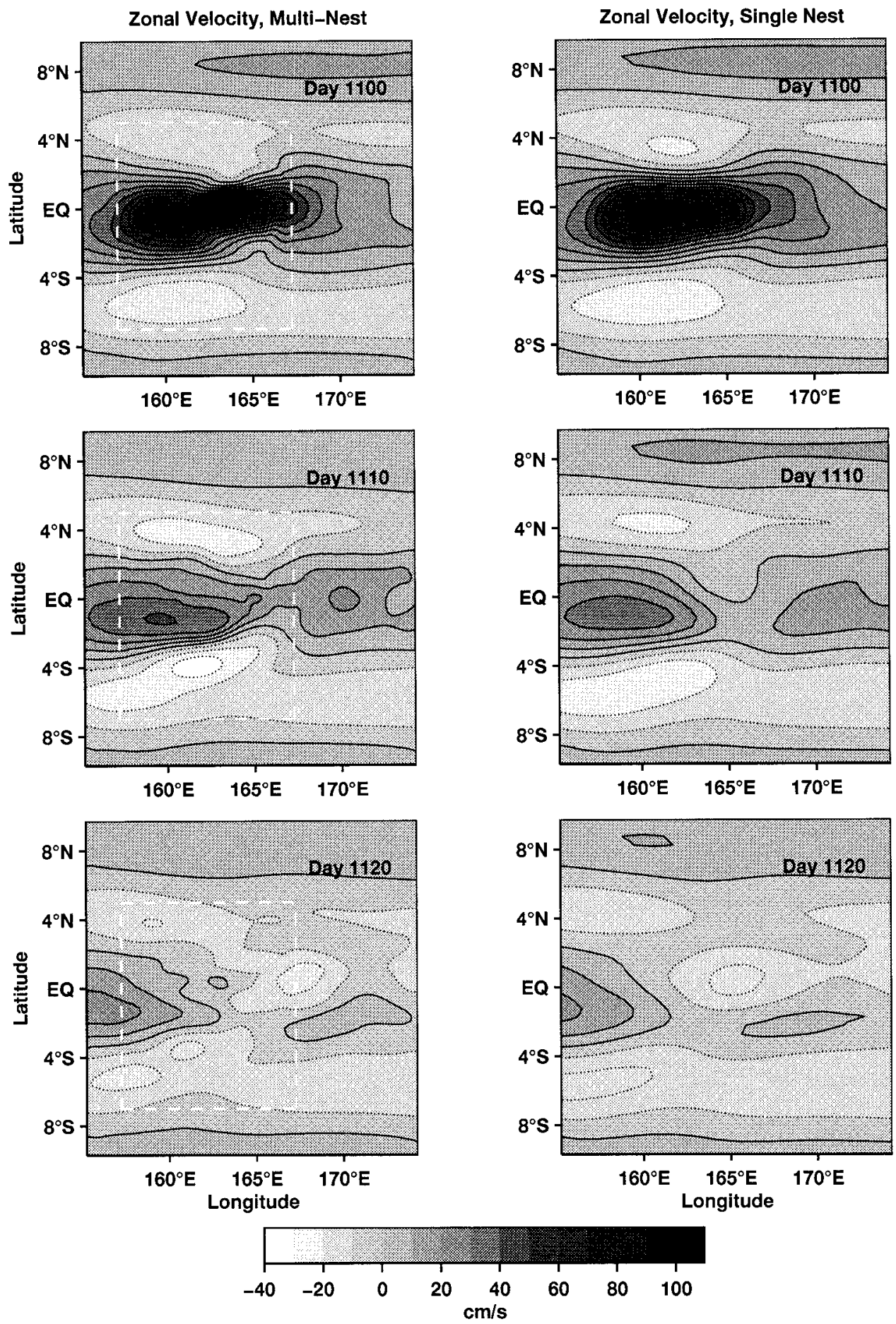

FIG. 15. The WWB experiment. Surface zonal velocity in the nested run (left panels) and single-mesh run (right panels). The area shown is for the region of the two inner meshes in the nested model.

cific. For this simulation, we select the real case of Tropical Cyclone Yuri in 1991 which, during the period from November 21 to November 25, remained almost at the same location and developed rapidly from a tropical depression to typhoon strength. This case has been well documented recently by Kindle and Phoebus (1995), using the U.S. Navy's operational global data assimilation system. Based on their data, we specify the wind stress radial profile using the linear formula first introduced by Chang and Anthes (1978) 

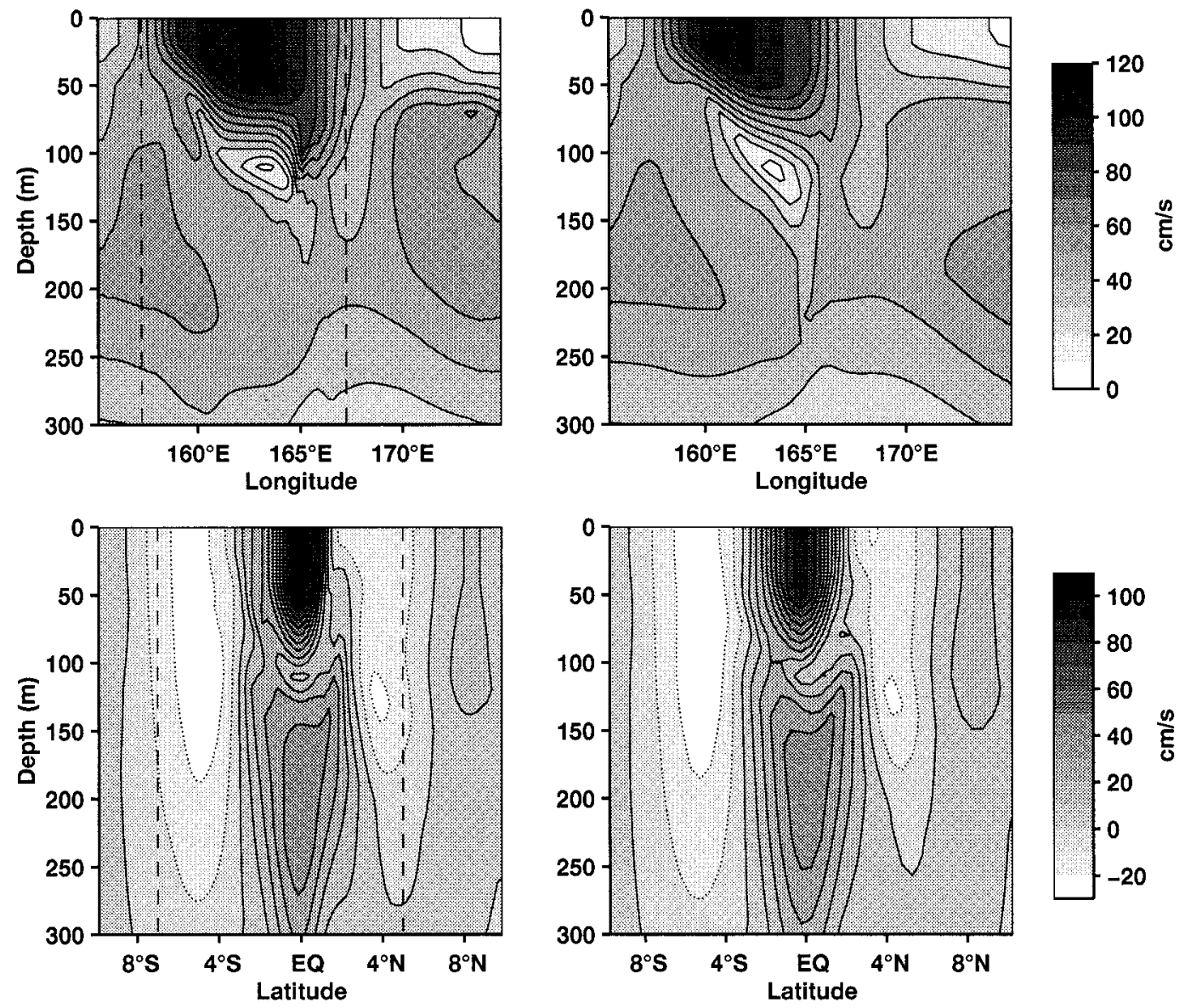

FIG. 16. The WWB experiment. Zonal (along the equator) and meridional (along $163.4^{\circ} \mathrm{E}$ ) cross sections of the zonal velocity at day 1100 for the nested run (left panels) and the single-mesh run (right panels).

$$
\tau_{0}^{\varphi}=\tau_{0 \max }^{\varphi} \begin{cases}\frac{r}{r_{m}}, & 0<r<r_{m} \\ \frac{r_{0}-r}{r_{0}-r_{m}}, & r_{m}<r<r_{0},\end{cases}
$$

where $\tau_{0 \max }^{\varphi}=0.5 \mathrm{~Pa}$ is the maximum azimuthal component of the wind stress, $r$ is the radial distance, and $r_{m}=100 \mathrm{~km}$ and $r_{0}=600 \mathrm{~km}$ are the radii of the maximum wind and the outer edge of the typhoon, respectively. The cyclone center is located at $7.5^{\circ} \mathrm{N}$, $168^{\circ} \mathrm{E}$.

For this experiment, we use a triply nested grid configuration in which the geometry of the outermost mesh and the mesh resolutions are the same as in the WWB experiments discussed above. The two inner meshes cover the regions $0^{\circ}-14^{\circ} \mathrm{N}, 161^{\circ}-175^{\circ} \mathrm{W}$, and $3.5^{\circ}-$ $11.5^{\circ} \mathrm{N}, 164^{\circ}-172^{\circ} \mathrm{W}$, respectively. The model is initialized in the same manner as for the WWB experiment, using the fields generated by the 3-yr single-mesh spinup driven by annual mean surface wind stress and heat fluxes. The integration started on day 1090 and the cyclone forcing was applied for 4 days. The model run was then continued until day 1120 with no forcing as Yuri moved away from the equator.

Figure 17 shows the evolution of the SST, with velocity arrows superimposed, in the region covered by the two inner meshes. These fields are compared to a single-mesh, $1 / 2^{\circ}$ resolution run. The tropical cyclone generates an energetic cyclonic circulation in the upper ocean. It is accompanied by an SST decrease induced by vertical turbulent mixing. In addition, the storm excites near-inertia-gravity waves that propagate primarily toward the equator with an eastward group velocity. It can be seen that stronger currents and larger SST cooling are produced by the nested run. No grid-scale noise can be seen in either the temperature or velocity fields at the nested-model interfaces. Careful analysis of the results indicates that the impact of mesh nesting on the wave propagation is minimal.

The cyclonic circulation moves in the north-northwest direction due to the advection of planetary vorticity. A jet generated behind the cyclone is considerably weaker in the single-mesh model $\left(29.2 \mathrm{~cm} \mathrm{~s}^{-1}\right.$ versus $41.8 \mathrm{~cm} \mathrm{~s}^{-1}$ for the nested case at day 1110). By day 

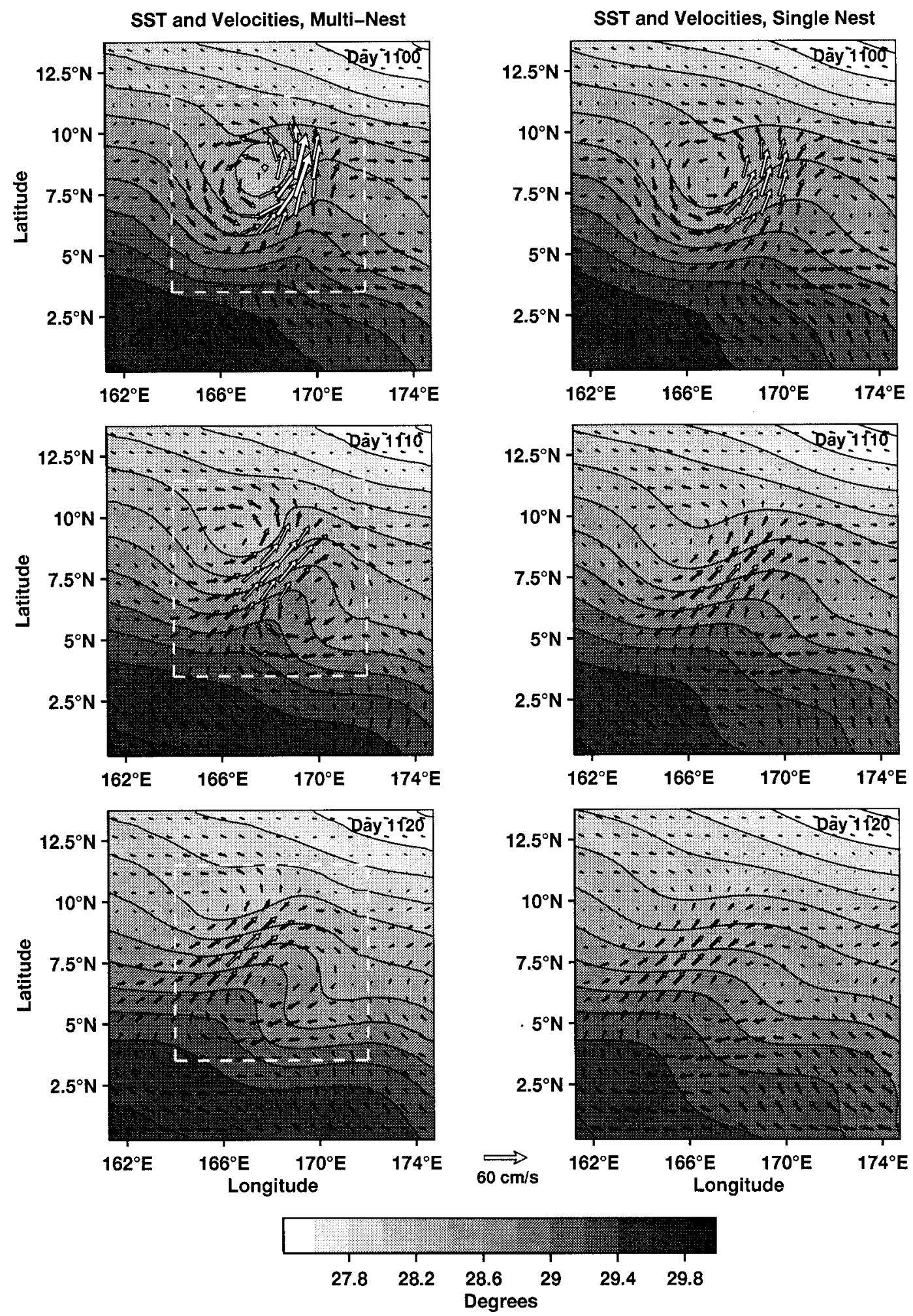

FIG. 17. Tropical cyclone experiment. Surface temperature and velocity fields for the nested run (left panels) and the single-mesh run (right panels). The area shown is for the region of the two inner meshes in the nested model. 
1110, an anticyclonic circulation has developed behind the cyclonic vortex in the nested model, but not in the single-mesh model. It is generated by the development of an annulus with a negative potential vorticity gradient. The low horizontal resolution in the single mesh is apparently not adequate to resolve this circulation. It is also seen that the finer grids in the nested model have allowed the development of much steeper SST gradients in the innermost mesh. By day 1120, the anticyclone has moved slowly to the west with a noticeable meridional twisting. The cyclonic vortex, however, has weakened considerably and stretched out in the zonal direction. This is because the evolution of both vortices is accompanied by Rossby wave radiation and nonlinear interaction with the generated Rossby wave wake. All these effects have been well investigated in the literature and it is not our intent here to present detailed analyses of the flow physics involved. We demonstrate here that the grid nesting has resulted in a much improved simulation of the current and temperature response to tropical cyclone forcing. This and the previous WWB experiment reveal the importance of increased model resolution in better determining the characteristics of the mesoscale ocean variability induced by strong synopticscale atmospheric forcing. Sufficient model resolution may be vital in some situations not only for resolving finescale features, but also for better representing the large-scale flow.

\section{Summary}

A multiply nested primitive equation ocean model has been described. The model employs a two-way interactive nesting technique originally proposed by Kurihara et al. (1979) and successfully applied for many years in the Geophysical Fluid Dynamics LaboratoryNOAA hurricane prediction model (Kurihara et al. 1998). One of the original features of the nesting scheme adopted in the model is that an interface where the two integration domains interact with each other (the dynamical interface) is intentionally separated from the mesh interface. This is done in order to prevent numerical noise from occurring at the mesh interface where noise may also result from the change of grid resolution. Furthermore, the interaction at the dynamical interface is expressed in the form of a flux condition so that the transports of mass, momentum, and heat do not fictitiously increase or decrease. Application of a time integration method that produces frequency-selective damping of waves, and the occasional use of spatial smoothing, effectively suppresses nonlinear computational instability in the model.

The model utilizes a sigma-coordinate system originally proposed by Gent and Cane (1989) and a hybrid mixed layer physics parameterization due to Chen at al. (1994a). Currently, the model invokes the reduced gravity approximation, in which the deep ocean is at rest and has a constant density. Efforts are underway to re- move this limitation by including a barotropic mode and bottom topography.

The results of various idealized experiments with the nested model were described. The nesting technique appears to be working very well. No distortion or computational noise associated with the dynamical interaction of adjacent meshes of differing resolution was found.

The formulation of the mesh nesting algorithm allows flexibility in deciding the number of meshes and the ratio of grid resolutions between adjacent meshes. The model has other advanced features as well, including realistic coastline geometry and spatially variable grid spacing, which we believe make it suitable for realistic simulations of the mesoscale dynamics of the sea. The model can also be used as a large-scale general circulation model (GCM) by using only one mesh. The most important feature of our nested model is the ability to combine large-scale and regional-scale predictions. It was demonstrated that the model can be integrated as a single-mesh GCM for long-term predictions, and the multinested structure can be introduced at any time for regional predictions in selected areas of interest or during unusual weather phenomena, such as tropical cyclones, wind bursts, etc. The model was tested as a GCM in a 3-yr spinup experiment of the large-scale circulation in the tropical Pacific Ocean. It demonstrated skill comparable to that of other recently developed GCMs. The resulting large-scale fields were then used in the nested configuration, as initial conditions for simulations of the ocean response to a westerly wind burst and a tropical cyclone. Significant improvements over a coarse singlemesh model have been achieved in resolving finescale features of the wind-induced current and temperature fields. These results highlight the importance of model resolution for realistic simulations of mesoscale ocean variability.

The usefulness and robustness of the developed nested-grid model will, no doubt, be determined from an accumulation of actual performance experience, in addition to the results of designed tests such as those presented in our paper. An effort is currently underway to apply the model to more realistic oceanographic and atmospheric situations.

Acknowledgments. This work was supported by the U.S. Department of Commerce National Oceanic and Atmospheric Administration through Grant NA46GPO187 and the U.S. Office of Naval Research through Grant N000149610758. The authors are grateful to Y. Kurihara and M. Bender at GFDL/NOAA for many fruitful discussions of the nested algorithm implemented in the present model and for providing access to the GFDL hurricane model code. Programming assistance from E. Foo is also gratefully acknowledged.

\section{REFERENCES}

Bender, M. A., R. J. Ross, R. E. Tuleya, and Y. Kurihara, 1993: Improvements in tropical cyclone track and intensity forecasts 
using the GFDL initialization system. Mon. Wea. Rev., 121 2046-2061.

Bryan, K., 1966: A scheme for numerical integration of the equations of motion on an irregular grid free of nonlinear instability. Mon. Wea. Rev., 94, 39-40.

Chang, S. W., and R. A. Anthes, 1978: Numerical simulations of the ocean's nonlinear, baroclinic response to translating hurricanes. J. Phys. Oceanogr., 8, 468-480.

Chen, D., L. M. Rothstein, and A. J. Busalacchi, 1994a: A hybrid vertical mixing scheme and its application to tropical ocean models. J. Phys. Oceanogr., 24, 2156-2179.

—, A. J. Busalacchi, and L. M. Rothstein, 1994b: The role of vertical mixing, solar radiation, and wind stress in a model simulation of the sea surface temperature seasonal cycle in the tropical Pacific Ocean. J. Geophys. Res., 99, 20345-20 359.

Flierl, G. R., 1987: Isolated eddy models in geophysics. Ann. Rev. Fluid Mech., 19, 493-530.

Fox, A. D., and S. J. Maskell, 1995: Two-way interactive nesting of primitive equation ocean models with topography. J. Phys. Oceanogr., 25, 2977-2996.

Gent, P. R., and M. A. Cane, 1989: A reduced gravity, primitive equation model of the upper equatorial ocean. J. Comput. Phys., 81, 444-480.

Ginis, I., M. A. Bender, and Y. Kurihara, 1997: Development of a coupled hurricane-ocean forecast system in the North Atlantic. Preprints, 22nd Conf. on Hurricanes and Tropical Meteorology, Ft. Collins, CO, Amer. Meteor. Soc., 443-445.

Gordon, C., and R. A. Corry, 1991: A model simulation of the seasonal cycle in the tropical Pacific Ocean using climatological and modeled surface forcing. J. Geophys. Res., 96, 847-864.

Harrison, D. E., and B. S. Giese, 1991: Episodes of surface westerly winds as observed from islands in the western Tropical Pacific J. Geophys. Res., 96, 3221-3237.

Hopfinger, E. J., and G. J. F. van Heijst, 1993: Vortices in rotating fluids. Ann. Rev. Fluid Mech., 25, 241-249.

Kindle, J. C., and P. A. Phoebus, 1995: The ocean response to operational wind bursts during the 1991-1992 El Nino. J. Geophys. Res., 100, 4893-4920.

Kurihara, Y., and J. L. Holloway, 1967: Numerical integration of a nine-level global primitive equation model formulated by the box method. Mon. Wea. Rev., 95, 509-530.

- , and G. J. Tripoli, 1976: An iterative time integration scheme designed to preserve a low-frequency wave. Mon. Wea. Rev. 104, 761-764.

_ and M. A. Bender, 1983: A numerical scheme to treat the open lateral boundary of a limited area model. Mon. Wea. Rev., 111, 445-453

— G. J. Tripoli, and M. A. Bender, 1979: Design of a movable nested-mesh primitive equation model. Mon. Wea. Rev., 107, 239-249

, C. L. Kerr, and M. A. Bender, 1989: An improved numerical scheme to treat the open lateral boundary of a regional model, Mon. Wea. Rev., 117, 2714-2722.

_ R. E. Tuleya, and M. A. Bender, 1998: The GFDL hurricane prediction system and its performance in the 1995 hurricane season. Mon. Wea. Rev., in press.

Larichev, V. D., and G. M. Reznik, 1976: Two-dimensional solitary Rossby waves. Dokl. Akad. Nauk SSSR, 231, 1077-1079.
Levitus, S., 1982: Climatological atlas of the world ocean. NOAA Prof. Pap. 13, U.S. Govt. Printing Office, 173 pp.

McPhaden, M. J., F. Bahr, Y. du Penhoat, E. Firing, S. P. Hayes, P. P. Niiler, P. L. Richardson, and J. M. Toole, 1992: The response of the western Equatorial Pacific ocean to westerly wind bursts during November 1989 to January 1990. J. Geophys. Res., 97, 14 289-14303.

Mellor, G. L., 1991: An equation of state for numerical models of oceans and estuaries. J. Atmos. Oceanic Techol., 8, 609-611.

Mesinger, F., and A. Arakawa, 1976: Numerical methods used in atmospheric models. Vol. 1. GARP Publ. Ser. No. 17, Joint Organizing Committee GARP, 64 pp.

Oey, L.-Y., and P. Chen, 1992: A nested grid ocean model with application to the simulation of meanders eddies in the Norwegian Coastal Current. J. Geophys. Res., 97, 20063-20086.

Philander, S. G. H., T. Yamagata, and R. C. Pacanowski, 1984: Unstable air-sea interactions in the tropics. J. Atmos. Sci., 41, 604613.

- W. J. Hurlin, and A. D. Seigel, 1987: Simulation of the seasonal cycle of the tropical Pacific Ocean. J. Phys. Oceanogr., 17, 1986-2002.

Price, J. F., R. A. Weller, and R. Pinkel, 1986: Diurnal cycling: Observations and models of the upper ocean response to diurnal heating, cooling, and wind mixing. J. Geophys. Res., 91, 84118427.

Robinson, A. R., Ed., 1983: Eddies in the Marine Science. SpringerVerlag, 609 pp.

Rossow, W. B., and R. A. Schiffer, 1991: ISCCP cloud data products. Bull. Amer. Meteor. Soc., 72, 2-20.

Seager, R., S. E. Zebiak, and M. A. Cane, 1988: A model of the tropical Pacific sea surface temperature climatology. J. Geophys. Res., 93, 1265-1280.

Smagorinsky, J., 1963: General circulation experiments with primitive equations: I. The basic experiments. Mon. Wea. Rev., 91, 291-304.

Spall, M. A., and W. R. Holland, 1991: A nested primitive equation model for oceanic application. J. Phys. Oceanogr., 21, 205-220.

Sutyrin, G. G., J. S. Hesthaven, J. P. Lynov, and J. J. Rasmussen, 1994: Dynamical properties of vortical structures on the betaplane. J. Fluid Mech., 268, 103-131.

UNESCO, 1981: Tenth report of the joint panel on oceanographic tables and standards. UNESCO Tech. Papers in Marine Sci. 36, 25 pp.

Webster, P. J., and R. Lukas, 1992: TOGA COARE: The Coupled Ocean-Atmosphere Response Experiment. Bull. Amer. Meteor. Soc., 73, 1377-1416.

Wyrtki, K., and B. Kilonsky, 1984: Mean water and current during the Hawaii-to-Tahiti Shuttle Experiment. J. Phys. Oceanogr., 14, 242-254.

Yoshida, K., 1959: A theory of the Cromwell current (the equatorial undercurrent) and of the equatorial upwelling. J. Oceanogr. Soc. Japan., 15, 159-170.

Zhang, D., H. Chang, N. L. Seaman, T. T. Warner, and J.M. Fritsch, 1986: A two-way interacting nesting procedure with variable terrain resolution. Mon. Wea. Rev., 114, 1330-1339.

Zhang, R.-H., and M. Endoh, 1992: A free surface general circulation model for the tropical Pacific Ocean. J. Geophys. Res., 97, $11237-11255$ 